Annales Geophysicae (2002) 20: 405-426 (c) European Geophysical Society 2002

\title{
Coordinated ground-based and Cluster observations of large amplitude global magnetospheric oscillations during a fast solar wind speed interval
}

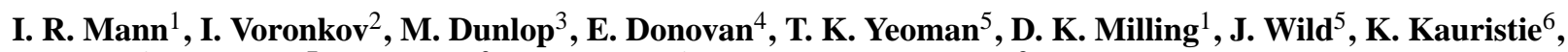

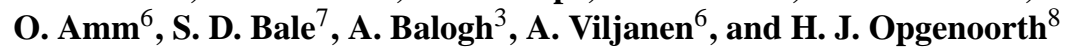 \\ ${ }^{1}$ Department of Physics, University of York, York, UK \\ ${ }^{2}$ Department of Physics, University of Alberta, Edmonton, Alberta, Canada \\ ${ }^{3}$ Imperial College, London, UK \\ ${ }^{4}$ Department of Physics and Astronomy, University of Calgary, Alberta, Canada \\ ${ }^{5}$ Department of Physics and Astronomy, University of Leicester, Leicester, UK \\ ${ }^{6}$ Finnish Meteorological Institute, Geophysical Research Division, P.O. Box 503, FIN-00101, Helsinki, Finland \\ ${ }^{7}$ Space Sciences Laboratory, University of California, Berkeley, USA \\ ${ }^{8}$ Swedish Institute of Space Physics, Uppsala Division, Sweden
}

Received: 18 June 2001 - Revised: 24 September 2001 - Accepted: 16 October 2001

\begin{abstract}
We present magnetospheric observations of very large amplitude global scale ULF waves, from 9 and 10 December 2000 when the upstream solar wind speed exceeded $600 \mathrm{~km} / \mathrm{s}$. We characterise these ULF waves using groundbased magnetometer, radar and optical instrumentation on both the dawn and dusk flanks; we find evidence to support the hypothesis that discrete frequency field line resonances (FLRs) were being driven by magnetospheric waveguide modes. During the early part of this interval, Cluster was on an outbound pass from the northern dusk side magnetospheric lobe into the magnetosheath, local-time conjugate to the Canadian sector. In situ magnetic fluctuations, observed by Cluster FGM, show evidence of quasiperiodic motion of the magnetosheath boundary layer with the same period as the ULF waves seen on the ground. Our observations represent the first simultaneous magnetometer, radar and optical observations of the characteristics of FLRs, and confirm the potential importance of ULF waves for magnetosphere-ionosphere coupling, particularly via the generation and modulation of electron precipitation into the ionosphere. The in situ Cluster measurements support the hypothesis that, during intervals of fast solar wind speed, the Kelvin-Helmholtz instability (KHI) can excite magnetospheric waveguide modes which bathe the flank magnetosphere with discrete frequency ULF wave power and drive large amplitude FLRs.

Paper submitted to the special issue devoted to "Cluster: First scientific results", Ann. Geophysicae, 19, 10/11/12, 2001.
\end{abstract}

Key words. Magnetospheric physics (magnetopause, cusp

Correspondence to: I. R. Mann (ian@ aurora.york.ac.uk) and boundary layers; MHD waves and instabilities; solar wind-magnetosphere interactions)

\section{Introduction}

Global oscillations of magnetospheric field lines can be excited in the magnetosphere by the solar wind. Dungey (1955) was the first to consider the possibility that ultra-low frequency (ULF) waves might constitute Alfvén waves which stand along geomagnetic field lines. Southwood (1974) and Chen and Hasegawa (1974) first considered how fast surface waves, excited by the Kelvin-Helmholtz instability (KHI) on the magnetopause, could drive standing Alfvén field line resonances (FLRs) on field lines of matching eigenfrequency. Following the observations of Kivelson et al. (1984), Kivelson and Southwood $(1985,1986)$ solved the problem of explaining the occurrence of discrete frequency FLRs by proposing that radially standing fast cavity mode waves could be excited between the magnetopause and a turning point inside the magnetosphere. Their model produced a discrete spectrum of cavity modes, each of which would resonantly drive a monochromatic FLR. Later work (see e.g. Samson et al., 1992; Walker et al., 1992; Wright, 1994) proposed that the outer magnetosphere might be better modelled as an open waveguide, rather than a cavity. In the waveguide model, discrete frequency wavegui de modes drive FLRs as they propagate and disperse down the waveguide, Mann et al. (1998) having presented the first observa- 
tional evidence for the existence and propagation of waveguide modes.

In recent theoretical work, Mann et al. (1999) showed how, under conditions of sufficiently fast magnetosheath flow speed, body type magnetospheric waveguide modes can be driven by magnetopause $\mathrm{KHI}$, in addition to the standard $\mathrm{KH}$ surface waves (see also Mills et al., 1999). Interestingly, the Mann et al. (1999) theory predicts that the conditions local to the magnetopause can control both the transport ULF wave power from the magnetosheath into the magnetosphere and the excitation of large amplitude FLRs. By examining the azimuthal phase speed characteristics of multiple harmonic FLRs, the Mann et al. (1999) theory can also be used to distinguish between FLRs driven by waveguide modes which result from solar wind impulses and those waveguide modes which are excited by magnetopause KHI (see Mann and Wright, 1999; Mills and Wright, and Mathie and Mann, 2000 , for more details).

To test this theory requires ground-satellite conjugate studies which compare the global structure of the FLRs seen on the ground with satellite measurements from close to the magnetopause. In this paper we conduct co-ordinated ground-based and Cluster observations of the global scale Pc5 ULF waves which are excited on the magnetospheric flanks between 9 and 10 December 2000 during an interval of fast $\left(>600 \mathrm{kms}^{-1}\right)$ solar wind speed. Our observations provide support for the hypotheiss that the KHI was responsible for exciting the large amplitude pulsations observed on the ground and for injecting significant energy into the flank magnetosphere. Our observations also provide further supporting evidence for the importance of ULF waves for magnetosphere-ionosphere coupling, in particular though the generation of auroral currents and the modulation of electron precipitation into the auroral ionosphere.

\section{Instrumentation}

We present co-ordinated ground-based and Cluster observations of ULF pulsations from 22:00 UT on 9 December (day of year 344) until 08:00 UT on 10 December 2000 (day of year 345). During this interval the magnetosphere was subject to a steady fast solar wind speed stream, the level $2 \mathrm{ACE}$ data from the SWEPAM (McComas et al., 1998) instrument (not shown) showing that the solar wind speed remained in excess of $600 \mathrm{~km} / \mathrm{s}$, the solar wind dynamic pressure remaining constant $\sim 2.5 \mathrm{nPa}$. Between approximately 22:00 UT and 02:00 UT, the Saskatoon SuperDARN radar (Greenwald et al., 1995), and CANOPUS (Rostoker et al., 1995) magnetometer and photometer data from the dusk local time sector showed clear evidence of the excitation Pc5 FLRs.

During December 2000, the Cluster spacecraft were still in the commissioning phase; however, data was being taken during certain intervals. Between approximately 22:00 UT and 24:00 UT, Cluster was local-time conjugate to the Canadian sector on an outbound trajectory from the northern dusk flank lobe into the magnetosheath. Figure 1 shows the po- sitions of the CANOPUS stations (red dots in the Canadian sector) and the field of view of the Saskatoon SuperDARN radar in the Canadian sector. Filled dots indicate the magnetic footprint of the Cluster 1 satellite trajectory, traced with the Tsyganenko 96 field model (Tsyganenko, 1996), plotted every $5 \mathrm{~min}$ and colour coded to indicate the radial position of the spacecraft. Similarly, un-filled dots show the radial projection of the trajectory of Cluster 1 and demonstrate the local time conjugacy of the Cluster satellites to the Canadian sector. The geographic pole is indicated with a cross; contours of geomagnetic latitude and longitude are plotted with increments of 10 and 15 degrees, respectively.

Later in this interval, from 01:30 UT until around 08:00 UT (and later), the morning local time sector showed clear evidence from European ground instrumentation of large amplitude pulsations and field line resonances. The field of view of the STARE Norway radar (belonging to the MIRACLE instrument network, Syrjäsuo et al., 1998), and the CUTLASS (Co-operative UK Twin Located Auroral Sounding System) Hankasalmi, Finland $\left(26.61^{\circ} \mathrm{E}, 62.32^{\circ} \mathrm{N}\right)$ SuperDARN radar, along with the positions of the IMAGE (Lühr et al., 1998) and SAMNET (Yeoman et al., 1990a) magnetometer stations (red dots in the European sector) used in this study are also indicated on Fig. 1. The geographic and magnetic coordinates of the CANOPUS and the IMAGE and SAMNET magnetometer stations are given in Tables 1 and 2 respectively. This configuration of ground-based instruments on the dawn and dusk flank, combined with data local to the dusk magnetopause from Cluster, allow us to present a detailed analysis of the energisation of both the dawn and dusk flank magnetosphere (e.g. McDiarmid et al., 1994) by the solar wind. The Cluster data allow us to test the hypothesis that fluctuations close to the magnetopause, such as the KHI, may have energised large amplitude pulsations and field line resonances on the flanks of the magnetosphere.

\section{Observations}

\subsection{Ground-based dusk sector observations}

We use Canadian sector ground-based data to identify and characterize a well-defined field line resonance and the associated wave dynamics in the dusk sector. Pc5 wave activity is evident in CANOPUS magnetometer data in the hours between 22:00 UT on 9 December and 04:00 UT on 10 December 2000. As we have supporting SuperDARN radar and CANOPUS photometer data, we focus on the latter part of this interval. Figure 2 shows the $H$ and $D$ components of the magnetic field measured by the CANOPUS magnetometer network from 00:00-04:00 UT, the data having been rotated from geographic $X$ and $Y$ polarisation into CGM $H$ and $D$ magnetic polarisation for the year 2000 epoch. Large amplitude Pc5 ULF wave activity is clearly visible throughout this interval in both the $H$ and $D$ components, with a particularly prominent wave packet being observed between 00:00 00:40 UT. Figure 2 also clearly shows that the ULF wave ac- 
Table 1. The coordinates of the CANOPUS magnetometer stations used in this study. The corrected geomagnetic coordinates (CGM) were calculated for the 2000 epoch at an altitude of $120 \mathrm{~km}$ using the International Geomagnetic Reference Field converter at http://nssdc.gsfc.gov/space/cgm/cgm.html

\begin{tabular}{|c|c|c|c|c|c|}
\hline \multicolumn{2}{|c|}{ Stations } & \multicolumn{2}{|c|}{ Geodetic } & \multicolumn{2}{|c|}{ CGM } \\
\hline Station & Code & Latitude & Longitude & Latitude & Longitude \\
\hline Taloyoak & TAL & 69.54 & 266.45 & 78.97 & 328.97 \\
\hline Contwoyto Lake & $\mathrm{CON}$ & 65.75 & 248.75 & 73.25 & 302.67 \\
\hline Rankin Inlet & RAN & 62.82 & 267.89 & 72.91 & 334.76 \\
\hline Eskimo Point & ESK & 61.11 & 265.95 & 71.20 & 331.90 \\
\hline Fort Churchill & $\mathrm{FCH}$ & 58.76 & 265.92 & 68.99 & 332.38 \\
\hline Fort Smith & FSM & 60.02 & 248.05 & 67.71 & 305.28 \\
\hline Rabbit Lake & RAB & 58.22 & 256.32 & 67.38 & 317.70 \\
\hline Fort Simpson & FSI & 61.76 & 238.77 & 67.53 & 292.55 \\
\hline Gillam & GIL & 56.38 & 265.36 & 66.69 & 331.95 \\
\hline Dawson & DAW & 64.05 & 220.89 & 66.00 & 272.23 \\
\hline Fort McMurray & MCM & 56.66 & 248.79 & 64.60 & 307.80 \\
\hline Island Lake & ISL & 53.86 & 265.34 & 64.26 & 332.32 \\
\hline Pinawa & PIN & 50.20 & 263.96 & 60.56 & 330.76 \\
\hline
\end{tabular}

Table 2. The coordinates of the IMAGE and SAMNET magnetometer stations used in this study. The corrected geomagnetic coordinates (CGM) were calculated for the 2000 epoch at an altitude of $120 \mathrm{~km}$ using the International Geomagnetic Reference Field converter at http://nssdc.gsfc.gov/space/cgm/cgm.html

\begin{tabular}{|c|c|c|c|c|c|c|}
\hline \multicolumn{3}{|c|}{ Stations } & \multicolumn{2}{|c|}{ Geographic } & \multicolumn{2}{|c|}{ CGM } \\
\hline Station & Code & Network & Latitude & Longitude & Latitude & Longitude \\
\hline Ny Ålesund & NAL & IMAGE & 78.92 & 11.95 & 76.08 & 112.21 \\
\hline Longyearbyen & LYR & IMAGE & 78.20 & 15.82 & 75.13 & 113.02 \\
\hline Hopen Island & HOP & IMAGE & 76.51 & 25.01 & 72.92 & 115.93 \\
\hline Bear Island & BJN & IMAGE & 74.50 & 19.20 & 71.34 & 108.87 \\
\hline Andenes & AND & IMAGE & 69.30 & 16.03 & 66.37 & 101.05 \\
\hline Troms $\varnothing$ & TRO & IMAGE & 69.66 & 18.94 & 66.55 & 103.59 \\
\hline Abisko & $\mathrm{ABK}$ & IMAGE & 68.35 & 18.82 & 65.21 & 102.41 \\
\hline Kilpisjärvi & KIL & IMAGE & 69.02 & 20.79 & 65.78 & 104.47 \\
\hline Masi & MAS & IMAGE & 69.46 & 23.70 & 66.07 & 107.09 \\
\hline Kevo & KEV & IMAGE & 69.76 & 27.01 & 66.20 & 109.92 \\
\hline Kiruna & KIR & IMAGE & 67.84 & 20.42 & 64.60 & 103.29 \\
\hline Muonio & MUO & IMAGE & 68.02 & 23.53 & 64.62 & 105.86 \\
\hline Pello & PEL & IMAGE & 66.90 & 24.08 & 63.45 & 105.55 \\
\hline Sodankylä & SOD & IMAGE & 67.37 & 26.63 & 63.81 & 107.89 \\
\hline Faroes & FAR & SAMNET & 62.05 & 352.98 & 60.71 & 78.02 \\
\hline Nordli & NOR & SAMNET & 64.37 & 13.36 & 61.42 & 95.44 \\
\hline Oulujärvi & OUJ & IMAGE & 64.52 & 27.23 & 60.88 & 106.72 \\
\hline Hankasalmi & HAN & IMAGE & 62.30 & 26.65 & 58.61 & 105.17 \\
\hline Nurmijärvi & NUR & IMAGE & 60.50 & 24.65 & 56.79 & 102.72 \\
\hline Uppsala & UPS & IMAGE & 59.90 & 17.35 & 56.44 & 96.37 \\
\hline Borok & BOR & SAMNET & 58.03 & 38.33 & 53.92 & 113.74 \\
\hline York & YOR & SAMNET & 53.95 & 358.95 & 50.88 & 78.97 \\
\hline
\end{tabular}

tivity is stronger over the eastern CANOPUS stations, the waves being much less apparent at the most (CGM) longitudinally distant stations DAW, FSM and FSI.

Figure 3 shows the (CGM) latitudinally stacked $H$ component power spectrum for the interval 00:00-01:00 UT, filtered between 1 and $10 \mathrm{mHz}$ (note that the DAW power spectra is not shown due to the low wave amplitudes and this station's large longitudinal separation from the other CANOPUS sites). Between the latitudes of the FCH and MCM stations, a very clear latitude independent spectral peak at 


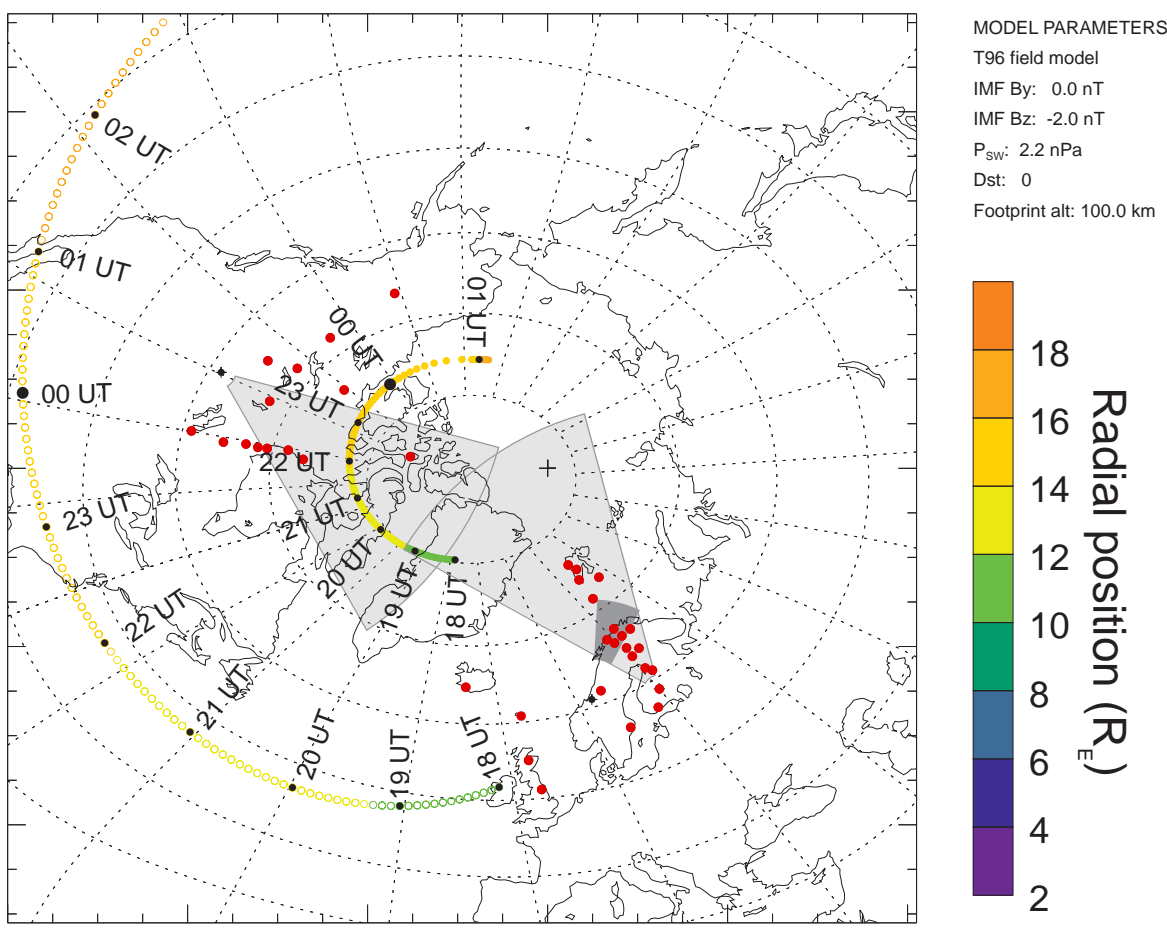

Fig. 1. Ground track of Cluster 1. Filled dots indicate the magnetic footprint, traced with the Tsyganenko (1996) field model, whilst unfilled dots indicate the radial projection of the Cluster 1 trajectory, colour coded with the satellites radial position. Also shown are the locations of the CANOPUS, IMAGE and SAMNET magnetometers, along with the fields of view of the CUTLASS Finland and Saskatoon SuperDARN radars, as well as that of the STARE Norway radar. around $3.0 \mathrm{mHz}$ is apparent, the half power points spanning approximately $2.8-3.4 \mathrm{mHz}$. In Fig. 4, we show the results from a complex demodulation analysis (e.g. Beamish et al., 1979) of this dominant spectral peak. The technique of complex demodulation allows one to determine the amplitude and phase characteristics of a non-stationary time series by comparison with a reference signal. Figure 4 shows the latitudinal variation of the $H$ and $D$ component amplitude (first panel) and phase (second panel) for the CANOPUS Churchill line at the time of maximum $H$ component amplitude ( 6 min period demodulate, bandpass filtered between $341 \mathrm{~s}$ and $381 \mathrm{~s}$ and analysed at 00:20 UT; see Mathie et al. (1999) for more details about applying the complex demodulation technique to FLRs).

For the dominantly Alfvénic signatures expected at a field line resonance, the magnetic perturbations in the magnetosphere are rotated through $90^{\circ}$ upon transmission through the ionosphere to the ground (e.g. Hughes and Southwood, 1976). Consequently, a well-developed FLR with a dominant toroidal polarisation in the magnetosphere would be rotated into a wave dominated by the $H$-component on the ground, exactly as is seen in Fig. 4. The $H$-component shows the clear latitudinal amplitude maxima and $180^{\circ}$ phase change expected for a well-developed field line resonance (see e.g. Mathie et al., 1999). The $D$-component also shows an amplitude maximum, and similarly displays a large phase change across the resonance.

The dominant wavepacket is best-defined in the $H$ component for the stations FSM, GIL and RAB. Using the maximum $H$-component demodulate from RAB and GIL gives a dimensionless azimuthal wavenumber $m \sim$
$0.5 \pm 0.3$ (the error, calculated from the CANOPUS sampling rate, probably gives an upper bound; using an average over the three maximum amplitude demodulates gives $m \sim 0.4 \pm 0.1$, the error being the standard deviation). Using the $D$-component, away from the resonance, can minimise any phase changes arising due to small latitudinal displacements between longitudinally spaced stations. Using the $D$-component station pair of ISL and MCM, away from the resonance, gives $m=+1.7 \pm 0.2$ (the $H$-component gives $m=+1.5 \pm 0.2$ ). Despite the slight variability, all these measurements are consistent with the expected eastward (tailward) propagation for dusk FLRs which are excited by waveguide modes driven by the solar wind. The low magnitude of $m$ is consistent with previous observations of FLRs which were believed to have been driven by magnetospheric waveguide modes (e.g. Fenrich et al., 1995; Mathie et al., 1999) and signifies that the waves are global scale magnetospheric phenomena.

In Fig. 5 we show in more detail the latitudinal dynamics of the pulsations along the Churchill line. The top panel shows a keogram of $557.7 \mathrm{~nm}$ data from the CANOPUS Rankin Inlet meridian scanning photometer (MSP) while the bottom panel shows a stack plot of the $X$-component magnetometer data from the six Churchill line magnetometer stations (AACGM, e.g. Baker and Wing, 1989, invariant latitudes from $65^{\circ}$ to $80^{\circ}$ ), plotted on the same amplitude scale and band-pass filtered between 60 and $660 \mathrm{~s}$. Both the magnetometer and MSP data show that Pc5 wave activity exists on magnetic latitudes up to Rankin Inlet. More interestingly, the phase of the $X$-component magnetic fields shows the clear polewards phase propagation which is expected for a 

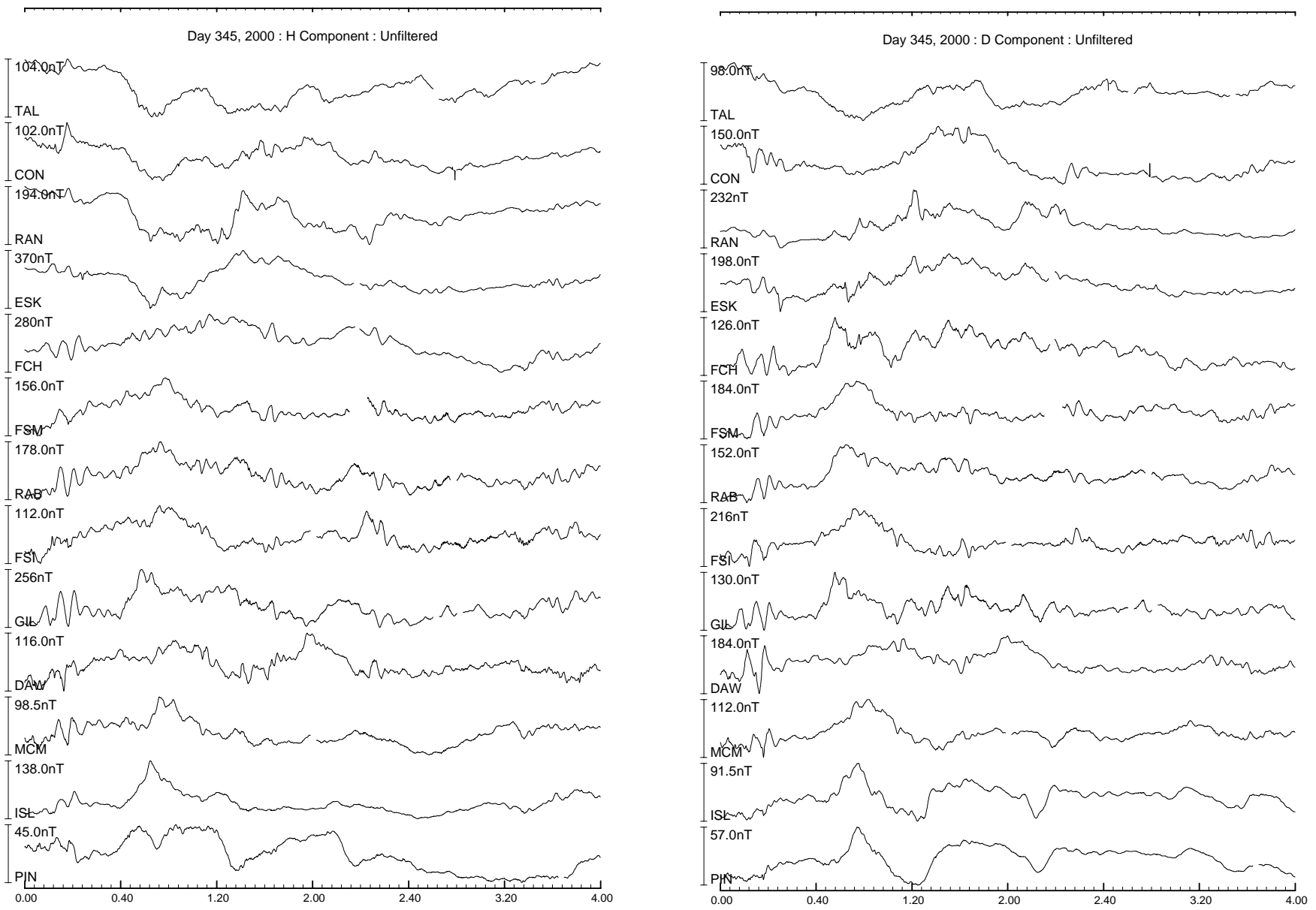

Fig. 2. Stacked raw $H$ (a) and $D($ (b) magnetograms from the CANOPUS magnetometer network between 00:00 UT and 04:00 UT on 10 December 2000.

driven field line resonance (e.g. Wright and Allan, 1996a).

The $630 \mathrm{~nm}$ data from the Rankin Inlet MSP (not shown) indicates that the boundary between open and closed fieldlines lies at $\sim 74^{\circ}$ AACGM invariant between 00:00 UT and 01:00 UT, after which it moves poleward (see Blanchard et al., 1997, for a discussion of the use of $630 \mathrm{~nm}$ data in the determination of the polar cap boundary). Hence, during the interval 00:00-01:00 UT, the peak $557.7 \mathrm{~nm}$ emissions are located roughly $2^{\circ}$ equatorward of the polar cap boundary (between the latitudes of the ESK and RAN magnetometer stations). This is consistent with Figs. 2, 3 and 5 which show that the highest latitude station TAL displays very little evidence of any clear ULF wave activity, consistent with its location on open field lines inside the polar cap. Moreover, the $630 \mathrm{~nm}$ data suggest that the latitude of the resonance maps into the central plasma sheet.

In Fig. 6 we show the ionospheric F-region plasma velocity as measured by beam 14 of the Saskatoon SuperDARN radar. Positive velocities correspond to motion towards the radar. As beam 14 of the radar passes over Fort Churchill (see Fig. 1 of Voronkov et al. (1997) for a schematic of relative positions of the SuperDARN field of view over the Churchill Line stations), positive velocities are indicative of south-west oriented plasma drifts. As can be seen in Fig. 6, there is a clear signature of a field line resonance in the radar data between 00:00 UT and 00:30 UT. Furthermore, comparing velocities measured in different beams, we can conclude that the resonance is dominantly polarised with the flow velocity in the azimuthal direction. Based on the SuperDARN data, the phase of the pulsations clearly increases with increasing latitude, again consistent with the poleward phase propagation seen in the $X$-component magnetometer data in Fig. 5.

Magnetometer and radar data are further analysed in Fig. 7 where the power spectral density of the $X$-component magnetic field from GIL, FCH, ESK, and RAN is shown in panel (a), and the power spectral density of plasma velocity from beam 14 of the Saskatoon radar is shown in panel (b). The data have been pre-filtered with a Butter filter with a low frequency cutoff at $0.5 \mathrm{mHz}$. The magnetometer data shows that the frequency of the spectral peak at each station decreases with latitude, again, as expected for a driven field line resonance. For a driven resonance, the response on any given field line will result from a combination of the fast mode driving frequency and the local natural Alfvén frequency of the field line (see e.g. ?, and references therein for a detailed discussion)McDiarmid++99. Once the driver has decayed, the 


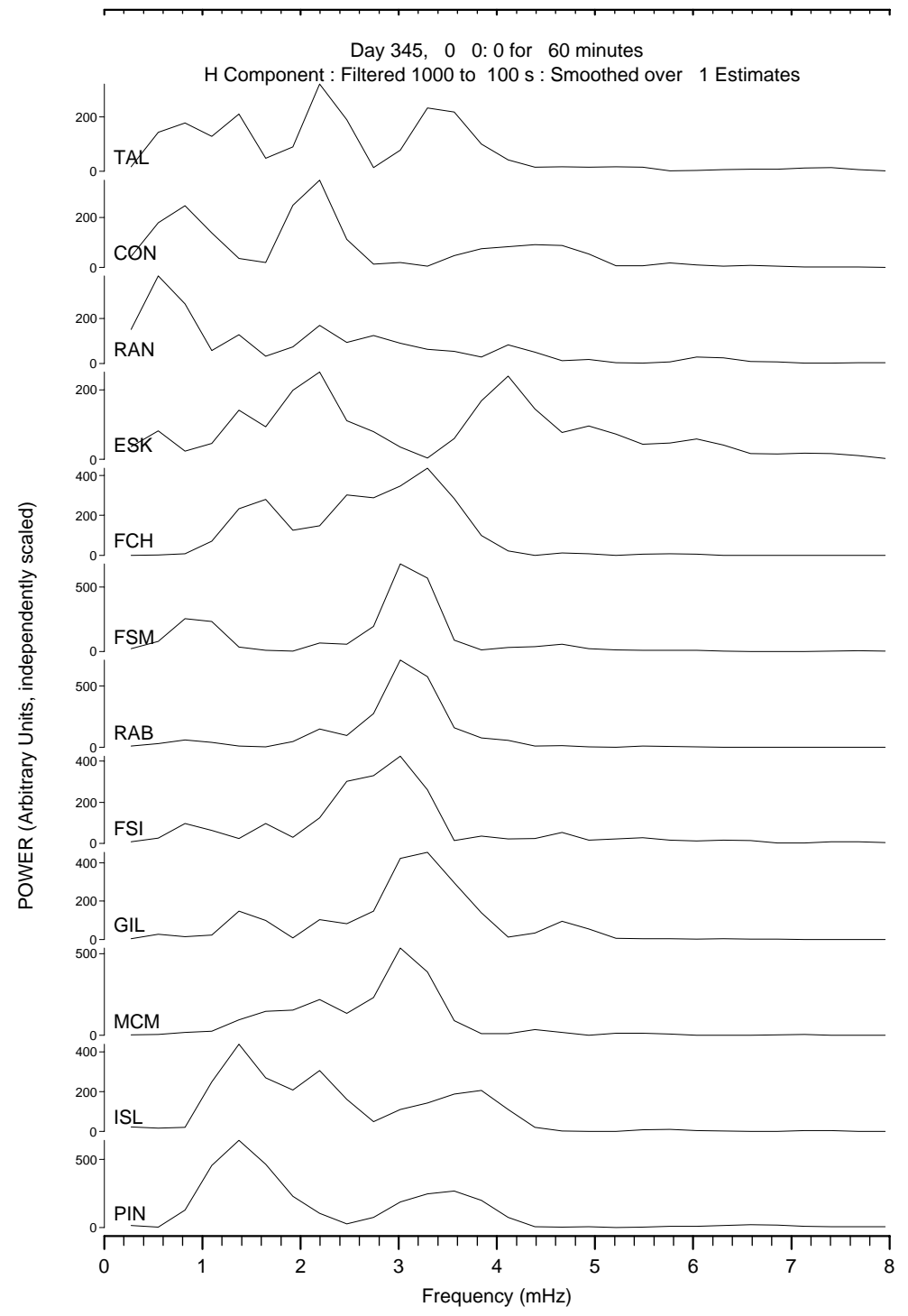

Fig. 3. CANOPUS stacked $H$-component power spectra (filtered from 1-10 mHz) from 00:00 UT to 01:00 UT on 10 December 2000.

field line resonance will be increasingly dominated by the response at the local Alfvén resonant eigenfrequency. This will result in phase mixing across the FLR (see e.g. Mann et al., 1995). The increase of wave period with latitude, characteristic of phase mixing, is the most expected and important feature of a well-developed field line resonance and is a direct consequence of an Earthward gradient of the Alfvén velocity in the magnetosphere outside the plasmapause. In panel (b), it can be clearly seen that the spectral distribution of the convection pulsations measured by the radar is close to the spectrum of magnetic pulsations registered by the Fort Churchill magnetometer, confirming that both instruments are measuring the same wave at the same location.

Observations of the field line resonance above are in very good agreement with theoretical expectations such as those presented Samson et al. (1998) which can be summarized as follows. At one phase of the resonance, the upward field aligned current (FAC) sheet is at the equatorward border of the resonance region. This FAC moves poleward, consistent with the latitudinal phase shift (c.f. Wright and Allan, 1996a and Mann, 1997). In the low- $m$ field line resonance, the upward FAC is mostly closed by a near-longitudinally directed Pedersen current. This in turn corresponds to an Eastward Hall current equatorward of the upward FAC sheet. The maxima of the Eastward Hall current occurs at the same time as the peak of the westward plasma convection flow. In Fig. 8, we combine the time dependence of the magnetic $X$-component Fort Churchill (normalized by $100 \mathrm{nT}$ ) and plasma velocities measured using beam 14 right above Fort Churchill. As seen from Fig. 8, these independent instruments show excellent agreement with both the magnetic and velocity measurements showing the same period and wavepacket structure. Moreover, the observed velocities and $X$-component magnetic field perturbations are clearly in 

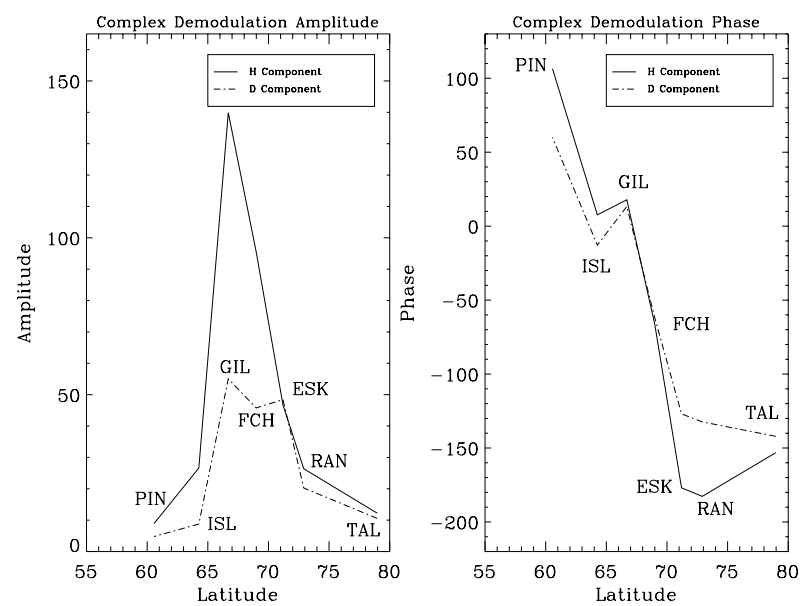

Fig. 4. Complex demodulation latitudinal amplitude (first panel) and phase (second panel) variations of the $H$ and $D$-components of the $2.8 \mathrm{mHz}$ wave along the CANOPUS Churchill line, centred on 00:20 UT.

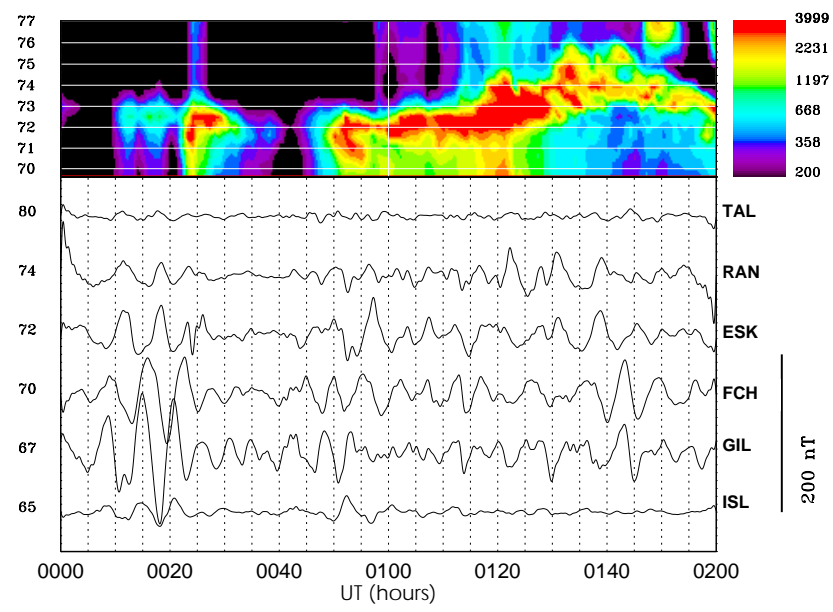

Fig. 5. CANOPUS pulsation activity between 00:00 UT and 02:00 UT on 10 December 2000: 557.7-nm emission intensity observed by the Rankin Inlet MSP as a function of AACGM invariant latitude (top panel) and stacked $X$-component magnetogram time series (bandpassed between 60-660 s) from the Churchill line magnetometers (bottom panel).

phase, as expected on the basis of the above arguments.

\subsection{Cluster dusk sector observations}

At 22:00 UT on 9 December, Cluster was on an outbound trajectory through the northern lobe in the dusk flank, conjugate to the Canadian sector on the ground (see Fig. 1). Figure 9 shows the trajectory of the four Cluster spacecraft in the GSE $X-Y$ plane, with blue dots marking the positon of Cluster 1 every hour. The spacecraft separations are increased by a factor of 20 to show their geometrical configuration and the position of Cluster at 22:00 UT is indicated. Between 22:00-24:00 UT Cluster remained close to constant $Z$ at around $Z=9 R_{E}$. The location of a model magne-
Date: 01210, SDARN Doppler Radar, Stn = Saskatoon, b14

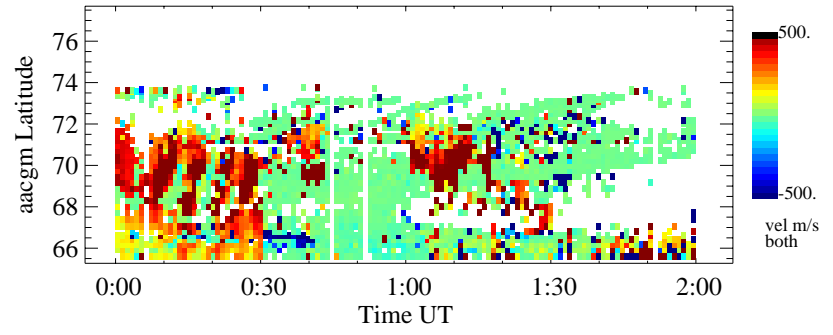

Fig. 6. Velocity in the ionospheric $F$-region along beam 14 of the SuperDARN Saskatoon radar between 00:00 UT and 02:00 UT on 10 December 2000. Positive velocities are in the direction toward the radar (close to the south-west direction above Fort Churchill).
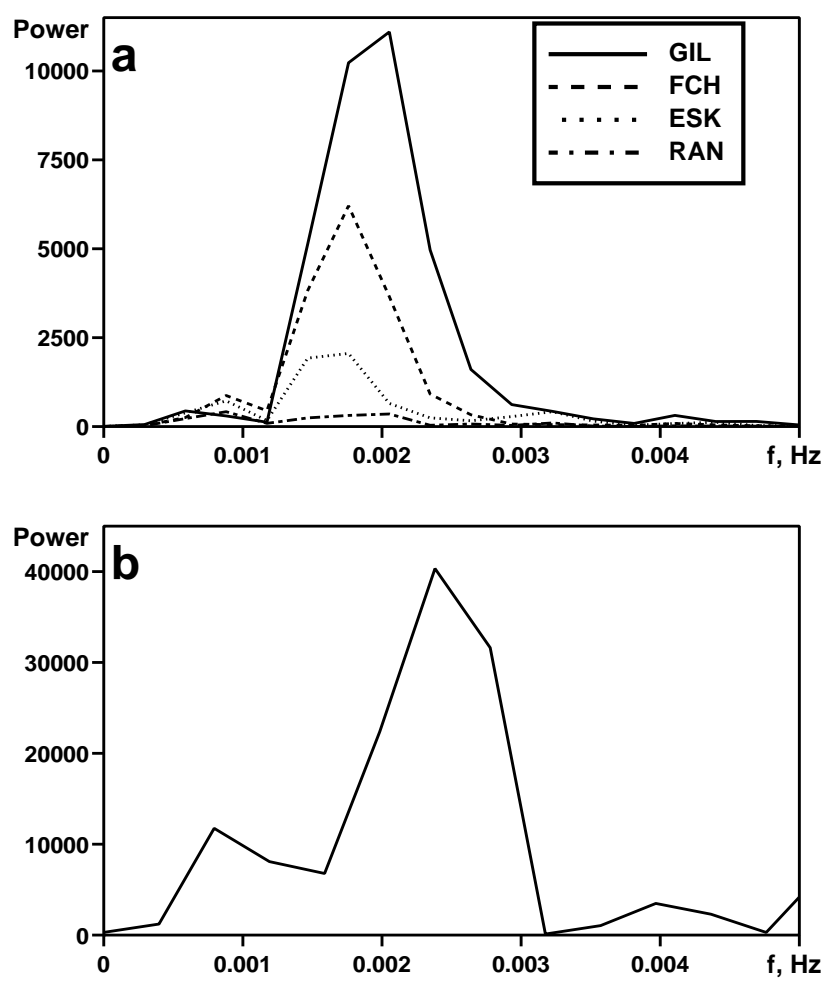

Fig. 7. Power spectral densities of the $X$-component magnetic field from selected magnetometer stations along the Churchill line (a), and from beam 14 of the Saksatoon SuperDARN radar at the range gate at $70^{\circ}$ above the Fort Churchill (FCH) magnetometer station (b). Spectra were taken for the interval 00:00 UT and 00:40 UT with an FFT, pre-filtered with a Butter filter with a cutoff at $0.5 \mathrm{mHz}$.

topause, for an upstream dynamic pressure of $3 \mathrm{nPa}$, is also shown in the GSE $X-Y$ plane at a fixed height of $Z=10 R_{E}$ (GSM). The vector direction of the magnetopause, normal (GSE) from the model at the location crossed by Cluster, is also indicated $\left(n_{M P}=(0.51,0.63,0.59)\right)$.

In Fig. 10 we show the magnetic field measured by the FGM instrument (Balogh et al., 2001) on-board Cluster 3 between 21:00 and 24:00 UT on 9 December 2000. The first, second and third panels show the spin resolution $4 \mathrm{~s}$ FGM magnetic field in the GSE $Z, Y$ and $X$ directions, respec- 


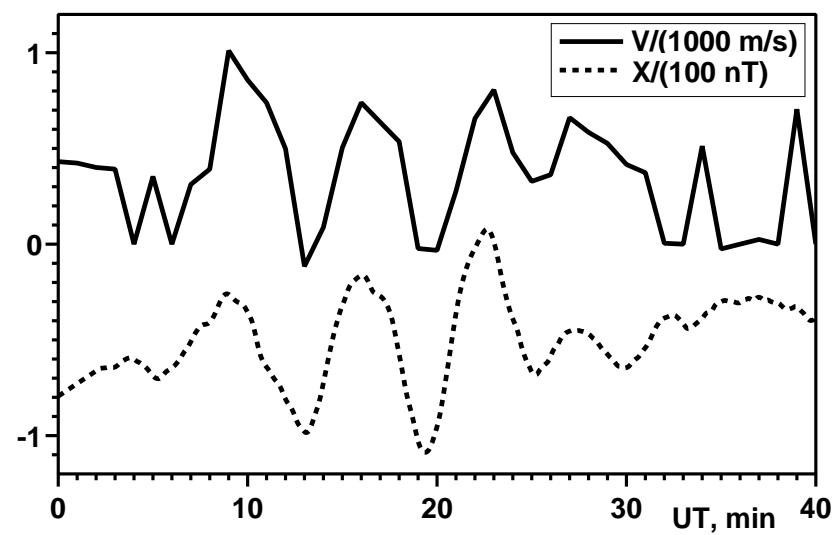

Fig. 8. Velocity (normalised by $1000 \mathrm{~m} / \mathrm{s}$ ) in the ionospheric $F$ region recorded by the Saskatoon radar (beam 14) at the latitude of Fort Churchill and magnetic $X$-component data (normalised by $100 \mathrm{nT}$ ) from Fort Churchill.

tively, with the final panel showing the magnetic field magnitude. As can be clearly seen, Cluster enters the magnetosheath boundary layer from the magnetosphere at 22:10 UT (first dotted red line, marked as BL). At 23:40 UT, Cluster crosses the magnetopause and enters the magnetosheath proper (second dotted red line, marked as MP). Between these times, Cluster travels deeper into the magnetosheath boundary layer, the magnetic field gradually decreasing from magnetospheric values (before 22:10 UT) towards the lower magnetosheath magnetic fields seen at the end of the interval (after 23:40 UT).

In Fig. 11 we show the results from a minimum variance analysis (Sonnerup and Cahill, 1967) of the magnetopause crossing around 23:40 UT which shows significant shear across the boundary. The top panel shows, after Dunlop et al. (1999), a scatter plot (pink crosses) of the magnetic field angles ( $\theta$ and $\phi$ GSE) measured by FGM on Cluster 2 between 23:34-23:48 UT. For a tangential discontinuity (TD) crossing, the field direction through the boundary should lie nearly on a plane (since $\boldsymbol{B} . \boldsymbol{n}=0$ ). The green curve represents a planar fit to the data points, indicating the field directions which would be expected if the magnetic field vectors during this magnetopause crossing lay in a plane. The red curve shows the locus of the plane which results from a minimum variance analysis (MVA) between these times, the minimum variance plane having a normal vector $n_{M V A}=(0.63,0.49,0.60)(\mathrm{GSE})$. The normal direction is stable and has an eigenvalue ratio in excess of 10 . There is excellent agreement between the fit to the observed data and the results from the minimum variance analysis indicating that the magnetopause was a fairly clean tangential discontinuity.

Interestingly, MVA analysis applied to Cluster 1 and Cluster 3 in the same interval produces normals which point in the same direction as Cluster 2 (Cluster $1 n_{M V A}=$ [0.64, 0.50, 0.59]; Cluster $\left.3 n_{M V A}=[0.64,0.51,0.58]\right)$. Cluster 4 produces a slightly different normal, tilting slightly

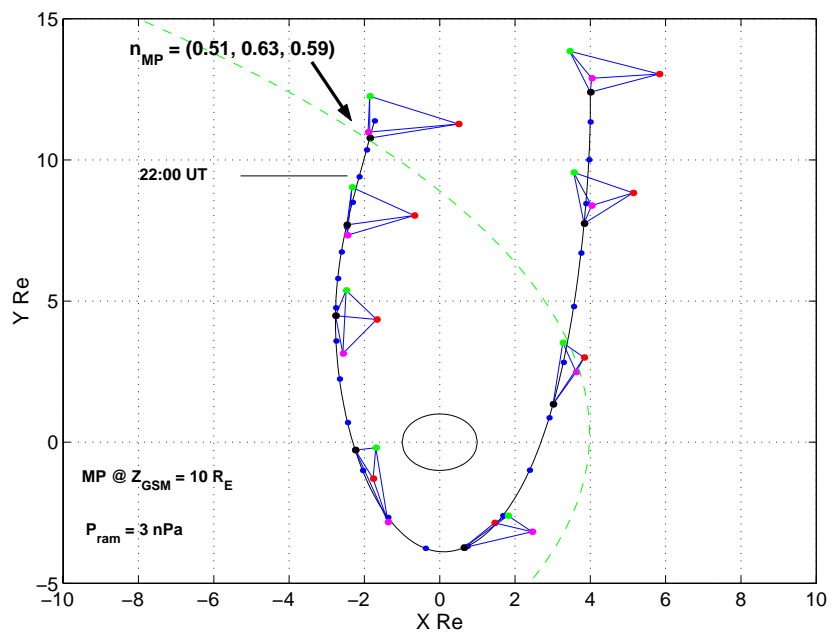

Fig. 9. Cluster trajectory in the GSE $X-Y$ plane, at a constant $Z$ GSM of $10 R_{E}$ on 9 December 2000 (black, red, green and magneta represent the Cluster 1, 2, 3 and 4 spacecraft respectively, and the spacecraft separations are magnified by a factor of 20). The position of a model magnetopause for a $3 \mathrm{nPa}$ dynamic pressure, and the location of Cluster 1 at 22:00 UT is also shown. Also indicated is the GSE vector direction of the model magnetopause normal ( $\mathrm{n}_{\mathrm{MP}}$ ) at the position where the Cluster trajectory crosses the model magnetopause.

in the $Z$ direction which may indicate some magnetopause compression $\left(n_{M V A}=[0.73,0.50,0.47]\right)$. However, in general, the normals from the MVA point approximately in the direction of the expected model MP normal direction (see Fig. 9). Consequently, the magnetopause crossing at 23:40 UT is consistent with the advection of a magnetopause which is planar on the scale of the Cluster spacecraft separation. Note, however, that the MVA determined normals point a little more sunwards than the direction predicted by the MP model. Interestingly, as pointed out by Otto and Fairfield (2000) (see also Fairfield et al., 2000, and references therein), if the magnetopause normal were being distorted slightly by the KHI, then for crossings from the magnetosphere into the magnetosheath (at the trailing edge), the magnetopause (MVA) normal would be expected to point slightly sunwards of the model MP normal, exactly as observed here.

The bottom panel of Fig. 11 shows the angle of the magnetic field out of the plane of the MVA normal, $\theta_{b n}$. It can be clearly seen that $\theta_{b n}$ is close to zero for the magnetopause crossing around 23:40 UT, as would be expected for a TD. Interestingly, the magnetic geometry of the entry of Cluster into the magnetosheath boundary layer around 22:10 UT is also well characterised by $\theta_{b n}$ angles which are close to zero. This suggests that the structure of the edge of the boundary layer was also planar, being aligned with a normal direction close to that of the magnetopause crossing at 23:40 UT. Unfortunately, this cannot be verified with a MVA since the entry into the magnetosheath boundary layer is characterised by low magnetic shear.

As Cluster enters the boundary layer, a number of interest- 
ing structures are revealed. Figure 12 shows the FGM magnetic field magnitude from the four Cluster spacecraft in the interval 22:00 to 24:00 UT. The first three panels show the data between 22:00-22:20 UT, 22:20-22:40 UT and 22:4023:00 UT, whilst the fourth panel summarises the Cluster FGM observations in the interval 22:00-24:00 UT. Again, the entry into the boundary later at 22:10 UT and the magnetopause crossing around 23:40 UT are both clearly seen. Following the entry into the boundary layer (BL) at 22:10 UT, the Cluster spacecraft measure magnetic field variations with short correlation lengths; different spacecraft measuring different field fluctuations. In agreement with the single spacecraft data shown in Fig. 10, there is a transition into a less turbulent and more magnetosphere-like region within the BL (with longer correlation lengths) between 23:18 and 23:40 UT, perhaps as a result of outwards motion of the magnetopause and boundary layer moving Cluster closer to the magnetosphere. After the magnetopause crossing at 23:40 UT, the correlation between individual Cluster spacecraft is again reduced, consistent with the Cluster spacecraft moving into the magnetosheath proper.

The fourth panel of Fig. 12 also highlights three particular regions of interest, each of which are magnified in panels one, two and three. In the first panel, all four Cluster spacecraft see effectively the same magnetic field as they travel through the outer lobe towards the magnetopause until around 22:10 UT. At this time, the four Cluster spacecraft enter the magnetosheath boundary layer (BL), each spacecraft entering the lower field magnitude region in the boundary layer at slightly different times. Spacecraft two (red) enters the BL first, with spacecraft one and three (black and green) crossing the BL next, followed by Cluster four (magenta). Looking again at the Cluster orbit configuration plot in Fig. 9, the ordering of the spacecraft entries into the BL can be understood on the basis of the orientation of the spacecraft with respect to the expected model magnetopause orientation. Figure 9 shows that in the $X-Y$ plane, Cluster 2 (red) is situated at highest GSE $X$ position, and would be expected to enter the BL first at this local time. Subsequent MP crossing would then be expected by Cluster 3 , followed by Cluster 1 and 4. However, Cluster 1, 3 and 4 do not lie at the GSE $Z$ position. In the northern lobe, the magnetopause will be inclined towards higher $Z$ at lower $Y$. Based on the Cluster orientation in the $Y Z$ plane, where Cluster 4 lies at the lowest position in $Y$ and $Z$, we would expect Cluster 1 and 3 to cross the MP before Cluster 4, exactly as observed.

Given that the low shear entry into the BL at 22:10 UT is characterised by MVA field angles $\theta_{b n}$ which are close to zero, the ordering of the spacecraft entries into the $\mathrm{BL}$ is also consistent with the hypothesis that the MVA normal from the 23:40 UT MP crossing also characterises the (presumably planar) geometry of the edge of the magnetosheath boundary layer. Hence we can conclude that the entry of Cluster into the magnetosheath BL at 22:10 UT can be explained by the motion of the edge of the locally planar BL across the spacecraft, whose normal lies close to the expected model magnetopause direction.
Examining the structure of the magnetosheath boundary layer between 22:10 UT and 23:00 UT shows that there are number of excursions of the Cluster spacecraft into regions characterised by low $|\boldsymbol{B}|$. Some of the entries into regions of lower magnetic field magnitude are observed by the Cluster spacecraft in the same sequence as that observed at the 22:10 UT entry into the magnetosheath BL (and that of the 23:40 UT magnetopause crossing) consistent with the advective motion of large scale approximately planar structures in the magnetosheath boundary layer across the spacecraft. For example, examining both Figs. 10 and 12, it is clear that, following entry into the BL at 22:10 UT, Cluster enters a low $|\boldsymbol{B}|$ region, the individual Cluster spacecraft maintaining approximately the same entry sequence as for the BL crossing at 22:10 UT before reaching the minima in $|\boldsymbol{B}|$ at around 22:13-22:14 UT. The subsequent motion back into a region of higher $|\boldsymbol{B}|$ broadly shows the reverse spacecraft ordering, although the signatures appear to be more complex. Other clear examples of entries into regions of low $|\boldsymbol{B}|$ with the same ordering can be seen beginning at around 22:23 UT, 22:36:30 UT, 22:43:30 UT and 22:49 UT, the minima in $|\boldsymbol{B}|$ observed by Cluster 3 being marked by green solid vertical lines in Fig. 10.

We believe that these regions of low $|\boldsymbol{B}|$ are observed by Cluster as a result of the advection of the magnetosheath boundary layer across the spacecraft, resulting in the sampling of regions of lower magnetic field which lie closer to the magnetopause, although probably not resulting in the crossing of the magnetopause itself. Examining the entries of the Cluster satellites into regions of low $|\boldsymbol{B}|$ in the boundary layer appears to suggest a possible 6-7 min quasiperiodic sequence. There are minima in $|\boldsymbol{B}|$ around 22:13 UT, 22:24UT, 22:31 UT, 22:38 UT, 22:44 UT and 22:56 UT (cf the green solid vertical lines in Fig. 10). As shown in Fig. 10, the decrease in $|\boldsymbol{B}|$ is, in general, related to a decrease in magnitude of both $B_{X}$ and $B_{Y}$. There is also some evidence that this quasi-periodic series may continue with $|\boldsymbol{B}|$ and $B_{X}$ decreases also being observed around 23:02 UT, 23:08 UT and 23:16 UT. Interestingly, during the regions of low $|\boldsymbol{B}|$, there is the suggestion from the bottom panel of Fig. 11 that these may also be accompanied by rotations of the MVA angle $\theta_{b n}$ close to zero. This may be suggestive of motion of the Cluster satellites closer to the magnetopause within the BL.

The 6-7 min quasiperiodic sequence of entries into regions of low $|\boldsymbol{B}|$ between 22:13 UT and 22:56 UT does, however, appear to be interrupted by $|\boldsymbol{B}|$ dips missing at times around 22:18 UT and 22:50 UT. Interestingly, as shown in the four spacecraft data from Fig. 12, at 22:18 UT there is evidence of a classic nested partial entry into a region of lower $|\boldsymbol{B}|$. Here, Cluster 4 does not enter the low $|\boldsymbol{B}|$ region, whilst Cluster 1 and 3 appear partially to enter a lower $|\boldsymbol{B}|$ region; Cluster 2 enters furthest into the low $|\boldsymbol{B}|$ region. This would be consistent with smaller amplitude inward motion of the magnetosheath boundary layer and hence only a partial entry of the Cluster quartet into a region of lower $|\boldsymbol{B}|$. This might be expected immediately following entry into the BL when the spacecraft remain close to the magnetosphere and 

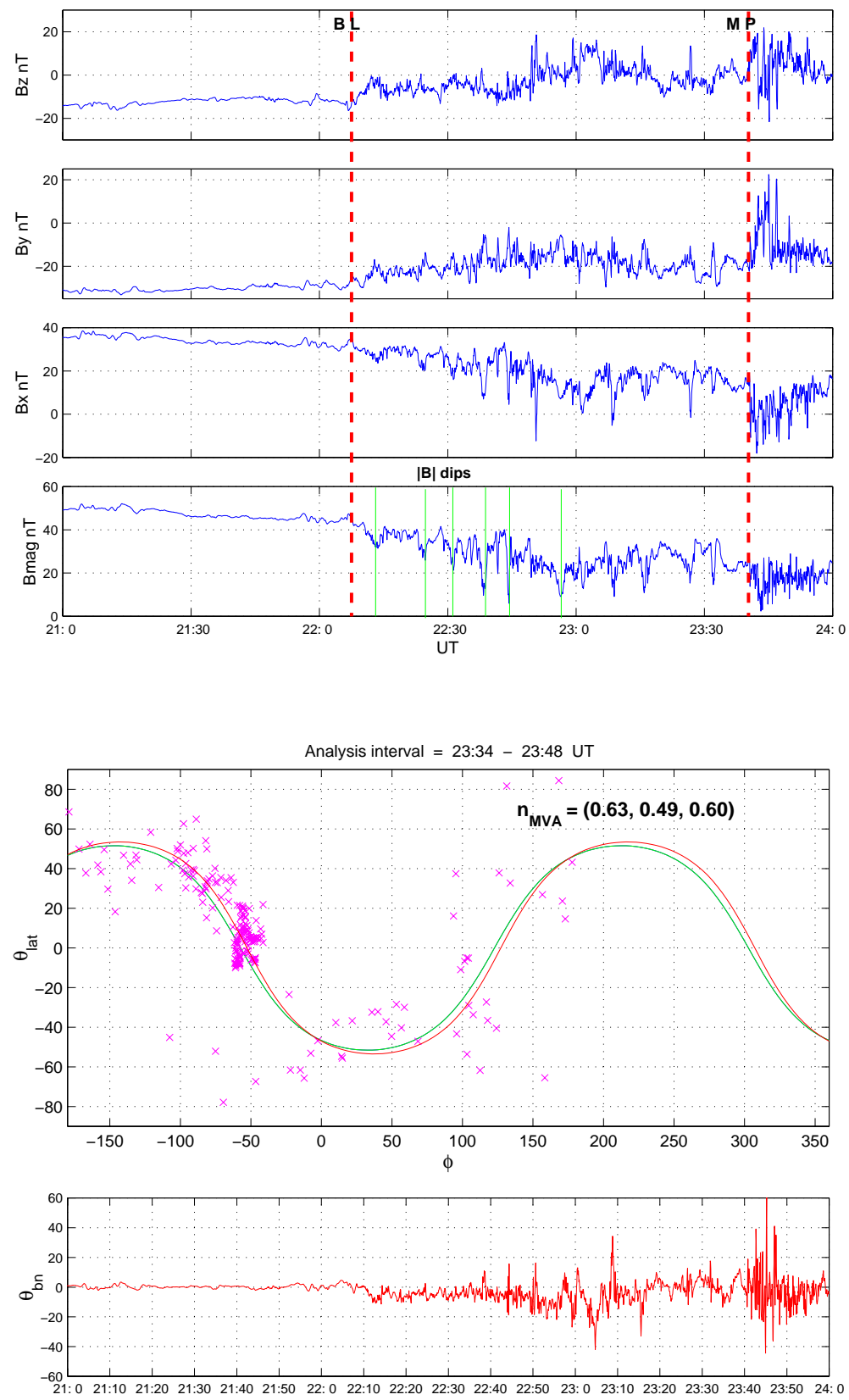

Fig. 10. GSE $B_{Z}, B_{Y}, B_{X}$ (first three panels), and $|\boldsymbol{B}|$ from Cluster 3 from 21:00 UT until 24:00 UT on 9 December 2000. Entry into the magnetosheath boundary layer (BL) at 22:10 UT and the magnetopause (MP) crossing around 23:40 UT are shown as red dashed lines, whilst excursions into regions of low $|\boldsymbol{B}|$ are indicated by green vertical lines in the final panel. See text for more details.

Fig. 11. (a) A scatter plot of the GSE magnetic field angles observed by Cluster 2 from 23:34-23:48 UT (pink crosses), and a best fit curve corresponding to a planar fit to the data (green curve) (top panel). Overplotted in red is the curve corresponding to a plane derived from a minimum variance analysis ( $\left.\mathrm{n}_{\mathrm{MVA}}\right)$ from the same interval. (b) The magnetic angle out of the minimum variance plane $\left(\theta_{b n}\right)$ observed by Cluster 2 for the interval 21:00-24:00 UT on 9 December 2000 (bottom panel). relatively far from the oscillating magnetopause. Certainly the observations in the interval 22:10-23:00 UT seem to be generally supportive of excurions into regions of increasingly lower $|\boldsymbol{B}|$ as Cluster travels deeper into the magnetosheath boundary layer. Similarly, at 22:49 UT there is evidence of sequential Cluster satellite motion into a slightly lower $|\boldsymbol{B}|$ region with the same ordering as the 22:10 UT entry into the BL. After 22:49 UT, as with the other entries into regions of low $|\boldsymbol{B}|$ in the quasi-periodic sequence, a clear decrease in $B_{X}$ is observed. However, in this case $|\boldsymbol{B}|$ does not decrease to such a low magnitude as that seen in the adjacent $|\boldsymbol{B}|$ dips around 22:44 UT and 22:56 UT. This may be in part because the change in $B_{X}$ is partially offset by an increase in $B_{Z}$. Consequently, the observations from both $22: 18 \mathrm{UT}$ and
22:49 UT can still be interpretted as being supportive of filling in the missing entries in the hypothesised 6-7 min quasiperiodic sequence.

This quasi-periodic motion of the magnetpause and boundary layers is consistent with that expected for a global mode of the magnetosphere, this global mode perhaps being excited by the development of the KHI on the dusk flank. For this explanation to be correct the KHI is required to have a wavelength which is much longer than both the Cluster spacecraft separation and the oscillatory displacement of the magnetopause, so that the local orientation of the magnetopause and boundary layer is approximately the same at each spacecraft and relatively close to the expected model MP orientation. As pointed out above, for crossings from the 


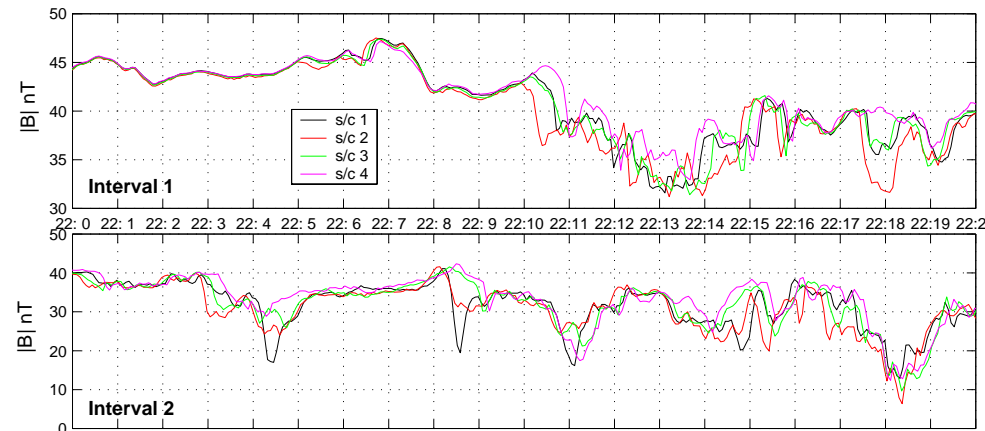

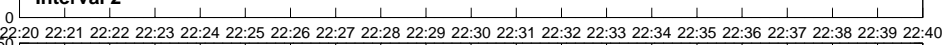

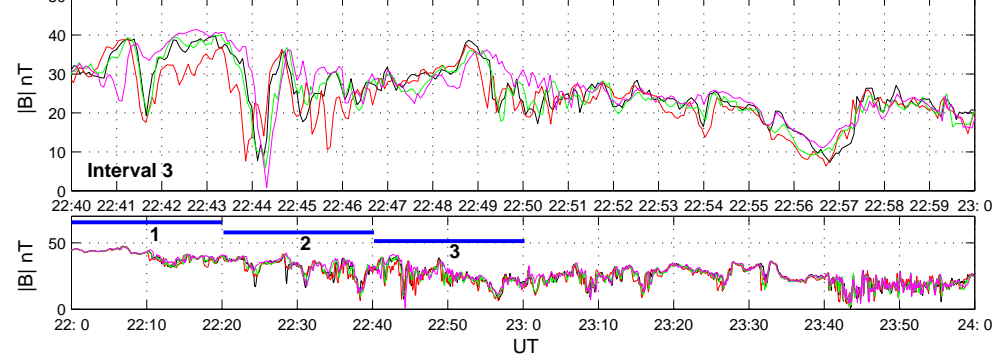

Fig. 12. Cluster four spacecraft measurements of $|\boldsymbol{B}|$ in the intervals 22:00-22:20 UT (first panel), 22:2022:40 UT (second panel) and 22:40 23:00 UT (third panel). The final panel shows a summary of the interval 22:00 24:00 UT; blue horizontal bars indicate the intervals expanded in the three panels above.
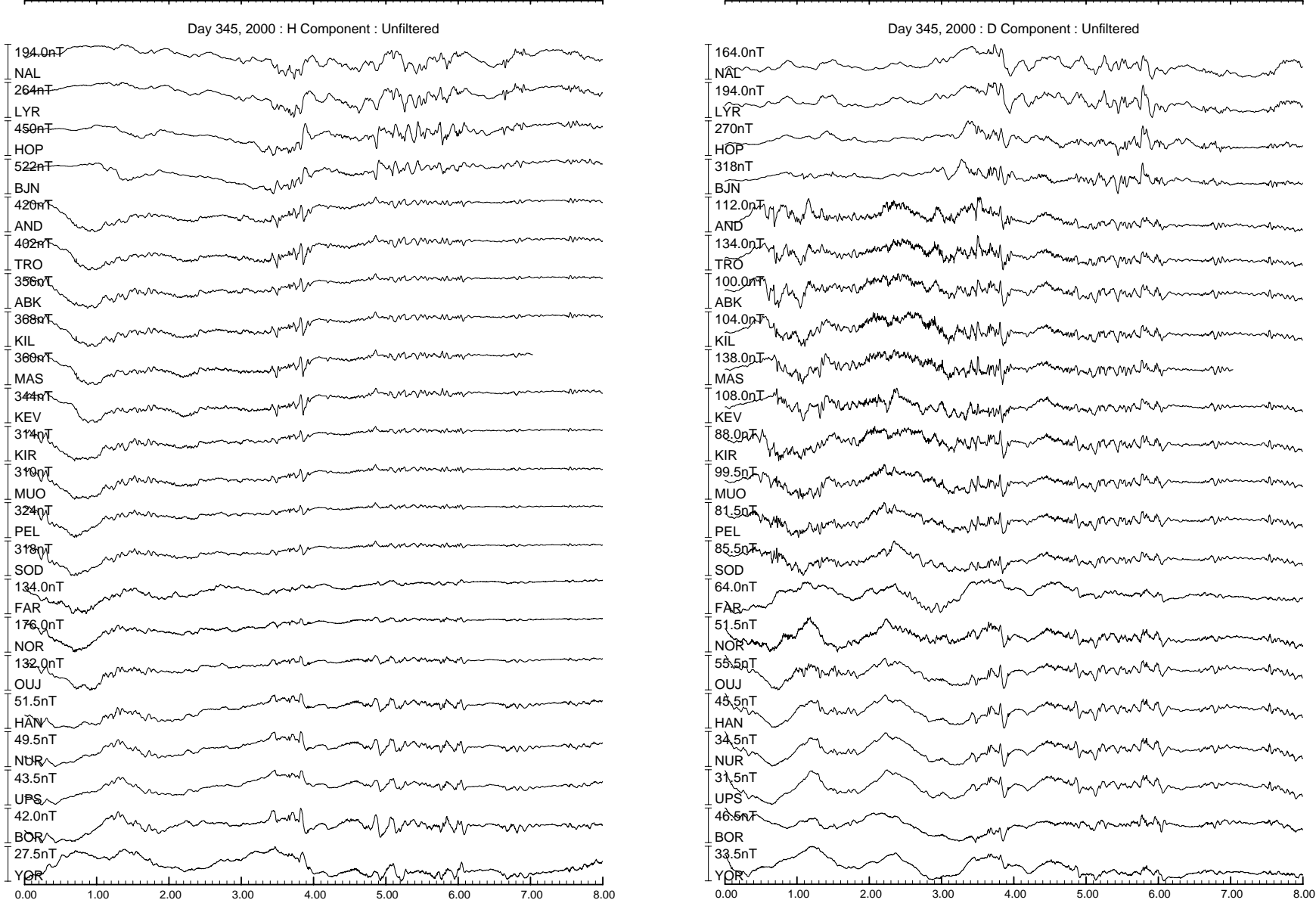

Fig. 13. Stacked $H$-component (a) and $D$-component (b) magnetograms from selected IMAGE and SAMNET magnetometer stations between 00:00 UT and 08:00 UT on 10 December 2000. Each magnetogram is independently scaled, the amplitude being indicated by the bars on the left hand side of each figure. 
magnetosphere into the magnetosheath, the KH would be expected to rotate the normal to point slightly sunwards of the model MP normal (cf. Fairfield et al., 2000); we discuss this further below.

There are also some partial entries of the Cluster spacecraft into lower $|\boldsymbol{B}|$ regions which do not fit the quasi-periodic sequence, such as that seen by Cluster 1 at 22:28:30 UT or the partially nested structure seen at 22:41:30 UT. However, these two cases may be the result of motion of the magnetopause due to changes in upstream ram pressure or result from local scale structures in the magnetosheath, rather than the development of long down-tail wavelength KHI. Indeed, both of these cases appear to represent situations where the Cluster satellites sample smaller $|\boldsymbol{B}|$ excursions than those adjacent $|\boldsymbol{B}|$ dips which do fit with the quasi-periodic sequence. Moreover, the observations from 22:28:30 UT show a case where Cluster 1 clearly samples the lowest $|\boldsymbol{B}|$ of all four Cluster satellites. Since Cluster 1 lies at the highest $Z$, this is consistent with the magnetopause and boundary layer moving towards lower $Z$ and flattening the magnetosphere and magnetosheath compared to both the model magnetopause shape and the normals inferred from the 22:10 UT and 23:40 UT BL and MP crossings. This would leave Cluster 1 sampling lower $|\boldsymbol{B}|$ as it "pokes out" further into the boundary layer as compared to the other Cluster satellites and could be consistent with distortion of the magnetopause and boundary layer on smaller spatial scales.

If the Cluster spacecraft are seeing periodic entries into low $|\boldsymbol{B}|$ regions as a result of the periodic motion of an approximately planar magnetosheath boundary layer and magnetopause, due to the action of the KHI, one might expect that there would be similar quasi-periodic entries into regions of higher $|\boldsymbol{B}|$ at the maxima of the outwards motion of the magnetopause. There are examples of transitions with exactly the reverse ordering to that observed for the BL entry at 22:10 UT (Cluster 4, then Cluster 1,3, and then Cluster 2), presumably as a result of the global outwards motion of the magnetopause and boundary layer. Indeed, as mentioned above, following the $|\boldsymbol{B}|$ dip at 22:13 UT there is clear outward motion with the expected ordering beginning at 22:14 UT. Similarly, the nested signature at 22:18 UT suggests clear inward then outward motion of the boundary layer. In a similar fashion, there is evidence of outward motion around 22:24:30 UT (following the inward motion at 22:23 UT); mid-way between the $|\boldsymbol{B}|$ dip at 22:31 UT and that at 22:38 UT there is another example of outward motion beginning at 22:34 UT. However, in this latter instance, the ordering of Cluster 1 and 2 is reversed from that expected for the motion of a planar boundary aligned with the magnetopause. This motion is immediately followed by what appears to be inward motion at 22:35 UT, where the Cluster spacecraft measurements are again ordered by the expected MP orientation. In general, the spacecraft ordering for outward motion of the boundary layer appears to be much more complicated than that for the inward motion. Often, for the outward motion of the boundary layer, the ordering seen by Cluster does not match that expected from the outward motion of planar structures whose orientation is determined by model magnetopause orientation. This suggests that the orientation of the magnetopause and magnetosheath boundary layer may differ between inbound and outbound motion. This could be explained by the development of $\mathrm{KH}$ vortex structures on the MP and in the BL; we discuss this further below.

In general the Cluster observations are consistent with quasi-periodic inward and outward motion of the magnetopause and magnetosheath boundary layer across the Cluster quartet. This quasi-periodic motion is what would be expected from a global magnetospheric oscillation such as a magnetospheric waveguide mode. Indeed, since the periodic magnetosheath motion has the same period as the ULF waves on the ground in Canadian sector, LT conjugate to Cluster (see Fig. 1), there is the strong suggestion that the motion of the magnetopause may have been related to driving the FLRs seen on the ground. It is possible that the development of a long wavelength KHI on the dusk flank caused the periodic global motion of the magnetosheath boundary layer across the Cluster quartet and that this KHI was the energy source for the large amplitude ULF waves seen on the ground. It should be noted, however, that any global compressional oscillations of the magnetosphere would be expected to cause inward and outward motion of the magnetopause. We discuss the possible solar wind energy sources for these global oscillations further below.

\subsection{Ground-based dawn sector observations}

During the interval 00:00-08:00 UT, on 10 December 2000 (DOY 345), ground-based instrumentation in the European sector also observed very large amplitude ULF waves as the Earth's rotation moved it into the dawn flank. Figure 13 shows the unfiltered $H$ (a) and $D$ (b) component magnetometer data from selected stations of the IMAGE and SAMNET magnetometer arrays (see Fig. 1 and Table 2 for details of the station locations). As can be clearly seen, very large amplitude ULF waves are prevalent throughout this interval across the whole European sector, with particularly clear wave packets between 00:45-02:00 UT, 03:30-04:00 UT and 04:45-06:10 UT.

Figure 14 shows the stacked $H$-component power spectrum, filtered between $100 \mathrm{~s}$ and $600 \mathrm{~s}$, for these IMAGE and SAMNET magnetometer stations between 00:45-02:15 UT. A sharp spectral peak, whose frequency is independent of latitude, can be seen at around $3.2 \mathrm{mHz}$, with a smaller subsidiary latitude independent peak at around $4.5 \mathrm{mHz}$ (this latter peak being less clear on the more westerly SAMNET stations during this interval).

In Fig. 15 we show the results from a complex demodulation analysis of the $3.2 \mathrm{mHz}$ spectral component from a latitudinal chain of selected magnetometer stations from the IMAGE array (bandpassed between $3.0 \mathrm{mHz}$ and $3.3 \mathrm{mHz}$ and taking an average of the two maximum $H$ amplitude demodulates from around 01:50 UT). Similar to the ULF waves 


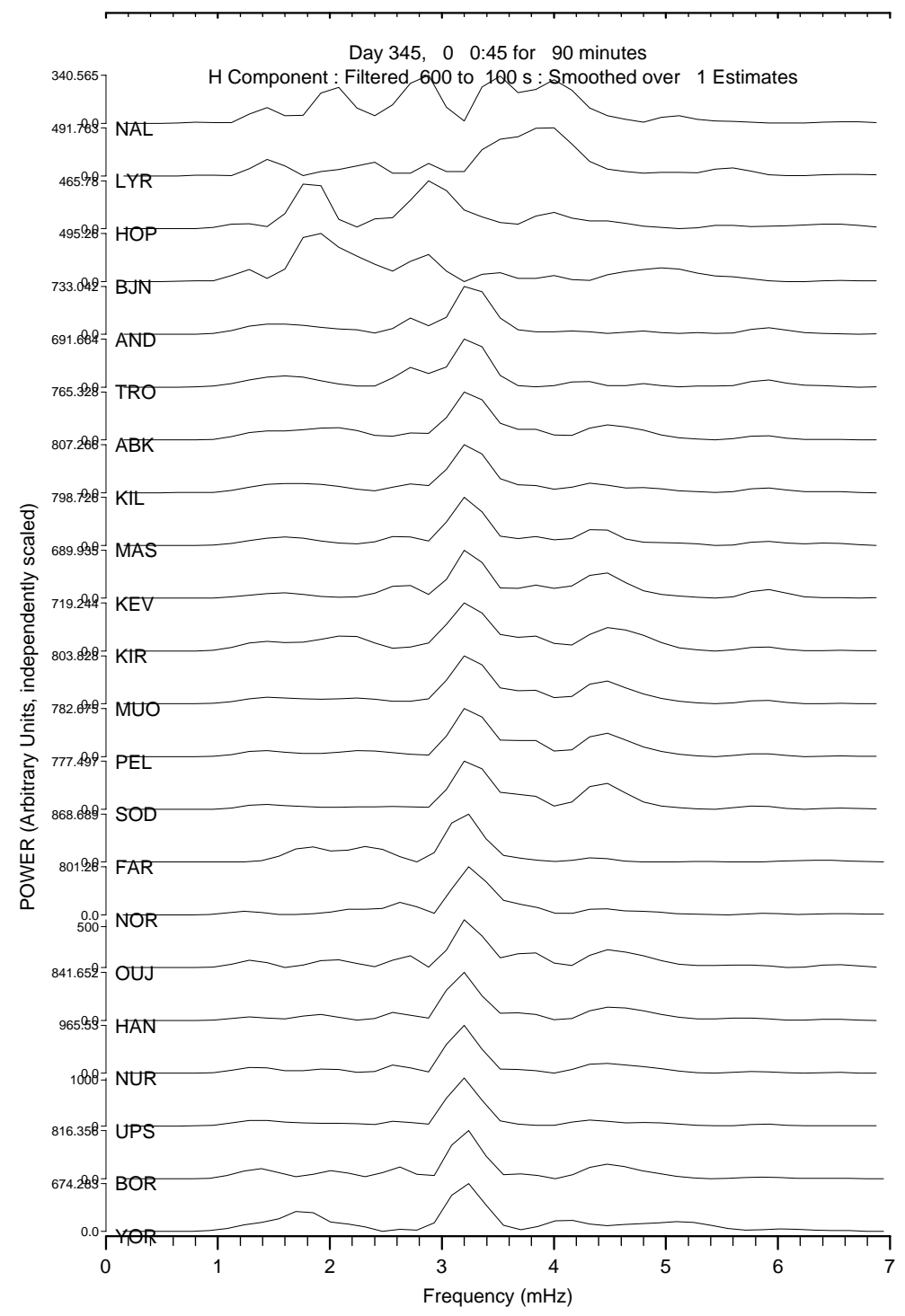

Fig. 14. Stacked $H$-component power spectra for the selected SAMNET and IMAGE magnetometer stations shown in Fig 14. Each power spectra is independently scaled. seen by CANOPUS on the dusk flank, the IMAGE magnetometers show these dawn flank waves to be dominanted by the $H$-component on the ground as expected for a dominantly toroidal wave in the magnetosphere. Again, the FLR characteristic is verified by the $180^{\circ}$ latitudinal phase change seen in the $H$-component. For this wave packet, the $D$ component also shows a latitudinal peak; however, there is a smaller phase change across the array in this component.

We can also estimate the azimuthal wavenumber of the waves by examining the phase changes at the longitudes local to the latitudinal chain of magnetometer stations shown in Fig. 15. Examining the phases from the complex demodulation analysis from Fig. 15 and using the $D$-component to minimise latitudinal phase changes, results in an $m$-value of $\sim-8.0 \pm 1.4$ from stations PEL and SOD. Closer to the resonance, the longitudinal chain KIL-MAS-KEV produces more variable phase estimates; however they are consistent with westward phase propagation with $m \lesssim 10$.
The clear ULF wave activity, seen by the IMAGE and SAMNET magnetometers, was also observed by the STARE VHF radar in the European sector. Figure 16 shows the radar back scatter power (top panel) and flow velocity (bottom panel) from beam 2 of the STARE Norway radar between 00:00 UT and 06:00 UT on 9 December 2000. In the backscatter power returns, very clear wave activity in the Pc5 band can be seen between 01:30-02:00 UT and between 03:30-04:00 UT, with smaller amplitude activity being observed throughout the interval from 00:30-04:00 UT. The wave activity is seen clearly in both the backscatter power and velocities between around 67 and 70 degrees geomagnetic, with evidence of the polewards phase propagation expected for a low- $m$ FLR (e.g. Wright and Allan, 1996a).

To examine the wave characteristics in more detail, we show in the top panel of Fig. 17 the backscatter power from selected range gates from beam 2. In this plot, very clear sinusoidal backscatter power oscillations are apparent, par- 

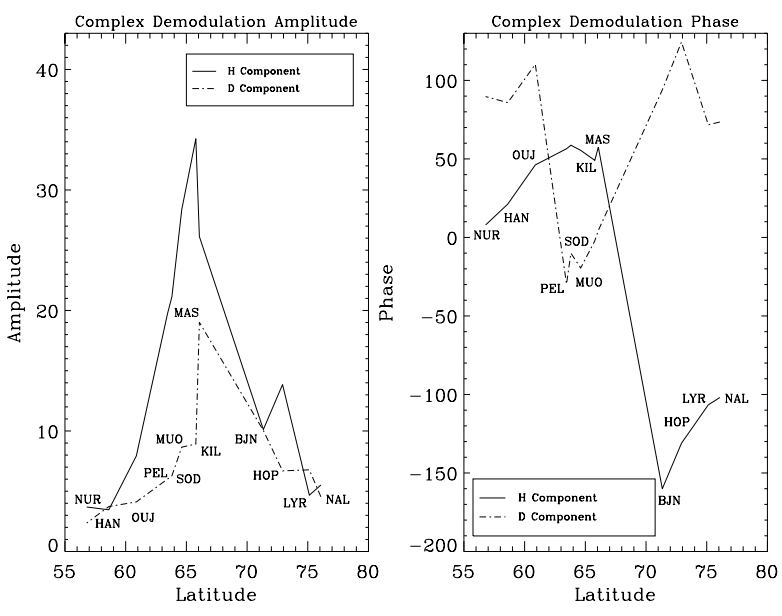

Fig. 15. Complex demodulation $H$ and $D$-component amplitude and phase for the $3.2 \mathrm{mHz}$ wave (average of 2 demodulates centred around 01:50 UT).

ticularly between 01:20-02:00 UT and 03:30-04:00 UT. The second panel in Fig. 17 shows the velocities seen by the CUTLASS Hankasalmi radar beam 5, close to the location of the STARE backscatter power returns.

At the beginning (before 00:30UT) and end (after 04:00 UT) of the interval shown in Fig. 16 there is no STARE radar backscatter, due to a lack of scattering irregularities resulting from the low ambient electric field (see e.g. Waldock et al., 1985). The VHF radar backscatter, seen by the STARE system in the centre of the interval, corresponds to an interval of enhanced ionospheric convection, as can be seen from the north-western pointing beam of the CUTLASS Hankasalmi radar in the bottom panel of Fig. 17 (see also Greenwald et al., 1995). The enhanced electric fields will produce backscatter through the two-stream instability (see e.g. Fejer and Kelly, 1980). The observed phase velocity of such backscatter is not a direct measurement of the line of sight component of the $\boldsymbol{E} \times \boldsymbol{B}$ velocity but is limited to the acoustic speed of the E-region ionosphere, although this itself increases non-linearly with the $\boldsymbol{E} \times \boldsymbol{B}$ velocity (Robinson, 1986). The enhanced convection electric field is then modulated by the ULF wave electric field, such that incomplete data coverage is available throughout the wave cycle, complicating any analysis of the wave (Yeoman et al., 1992). However the observed Doppler velocities are unidirectional and the backscatter intensity, which itself increases with $\boldsymbol{E} \times \boldsymbol{B}$ velocity, will thus provide a good proxy for the ULF wave amplitude (see Yeoman et al., 1990b; Shand et al., 1996).

To examine the properties of the ULF waves seen by STARE, Fig. 18 shows the unfiltered power spectrum of the backscatter power from beam 2 , range gate 38 , between 01:30-02:00 UT. A clear broad spectral peak, centred on $2.7 \mathrm{mHz}$, can be seen, in good agreement with the magnetometer observations presented in Fig. 14. In Fig. 19 we show the latitudinal amplitude and phase variation of the
$2.7 \mathrm{mHz}$ component from STARE beam 2 for range gates 28, 30, 32, 34, 36, 38, 40 (cf., the top panel of Fig. 17). A clear latitudinal amplitude maximum, at around $68.7^{\circ}$ geomagnetic, is seen with a phase change of around $50^{\circ}$ between 67.8 and $69.1^{\circ}$.

Clearly, there is excellent agreement between the wave activity seen by STARE and by the magnetometers. Both see a clear ULF wave with frequency close to $3.2 \mathrm{mHz}$ (note that the STARE radar spectrum in Fig. 18 has limited frequency resolution due to the analysis of only $30 \mathrm{~min}$ of data). The STARE data shows that the power in spectral peak at $2.7 \mathrm{mHz}$ demonstrates both the amplitude maxima and phase change expected from a FLR. Comparing Figs. 19 and 15, we see that the STARE inferred resonant latitude lies in the gap of magnetometer coverage between MAS and BJN (66$71^{\circ}$ geomagnetic). The resonant latitudes inferred from the IMAGE and STARE data appear to be entirely consistent with the limited latitudinal extent of the STARE backscatter, revealing part of the expected $180^{\circ}$ phase change which is seen over a wider latitudinal range by the magnetometers. Previous observations of Pc5 ULF waves have also placed the resonant field line of waves around $3 \mathrm{mHz}$ frequency between the Scandinavian mainland and BJN station (c.f., the 2.6 and $3.7 \mathrm{mHz}$ FLRs presented in Fig. 8 of Mathie and Mann, 2000).

We can also estimate the azimuthal wavenumber of the waves using the STARE observations in this interval. Since the STARE radar measures variations along the radar line of sight, calculating the $m$-values with STARE requires a careful consideration of the geometry of the differing look directions from beam to beam, which can influence the inferred $m$-values especially if the waves are not linearly polarised. Low- $m$ FLRs are, in general, elliptically polarised at resonance and this can cause the radar beam geometry to influence radar inferred $m$ (see e.g. Ziesolleck et al., 1998). The STARE data in the interval 01:30-02:00 UT suggest westwards phase propagation with an $m$-value $\sim 4$. This is consistent with the magnetometer estimates and, with a FLR being driven by a tailwards propagating source such as a magnetospheric waveguide mode, in the morning local-time sector. Moreover, this suggests that the STARE latitudinal phase profile shown in Fig. 19 is not significantly contaminated by the $3^{\circ}$ of longitude covered by the latitudinal range.

Returning to Fig. 13, we can see that large amplitude ULF wave activity continues thoughout the morning sector. Several other large amplitude wavepackets are apparent in both the $H$ - and $D$-components, the $H$-component being dominant in each wavepacket with the two wave packets between 03:30-04:00 UT and 04:45-06:10 UT being particularly clear. Amplitude and phase analysis of the 03:30 04:00 UT wave packet show that there is a clear spectral peak at around $2.5 \mathrm{mHz}$ however, the behaviour of the amplitude and phase at this frequency during this interval is complicated by the large amplitude impulsive signature at around 03:52 UT. This feature is clearly related to a poleward moving arc seen by the all-sky-cameras (ASC) of the MIRACLE network (not shown). The ASC camera, the magnetometer, 


\section{STARE PARAMETER PLOT}

10 Dec 2000: STARE Norway beam 2 [pwr \& vel]

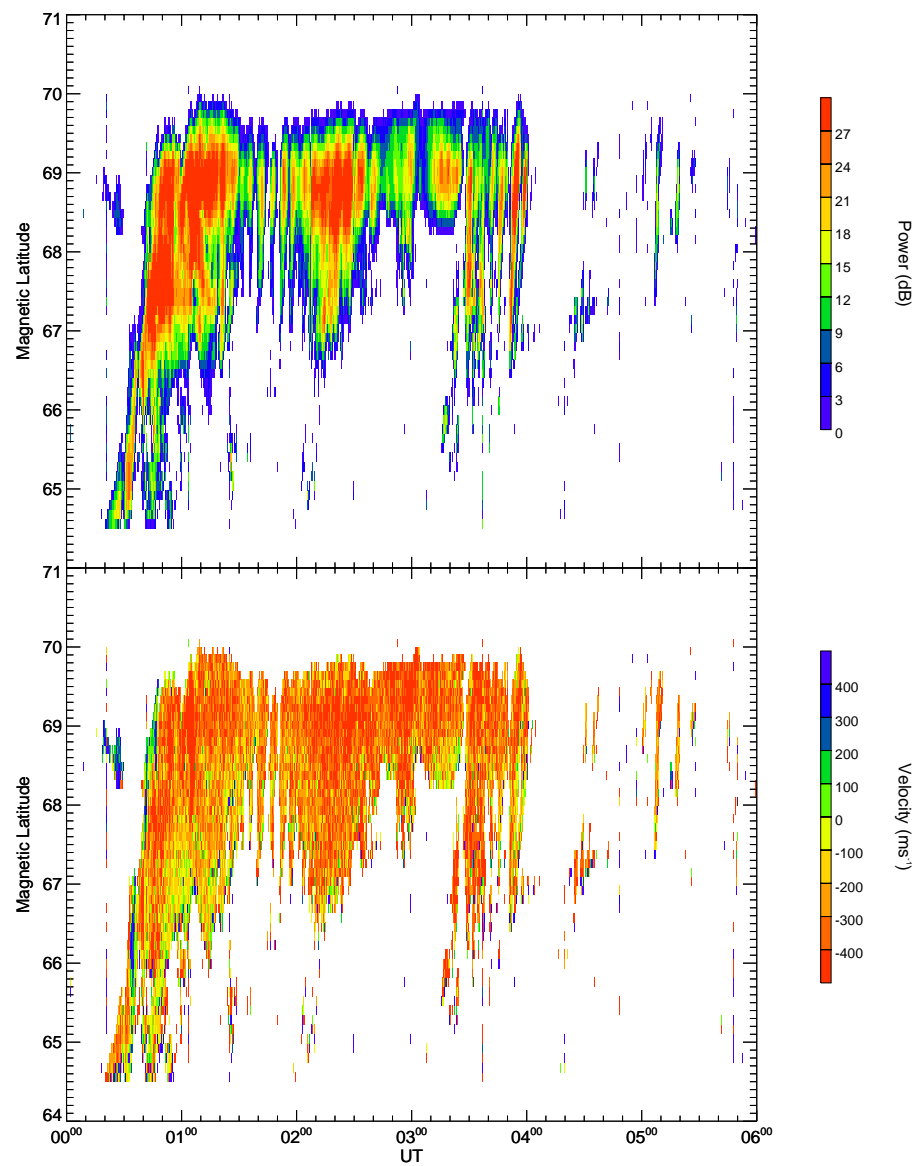

Fig. 16. Backscatter power (top panel) and velocity (lower panel) for the STARE Norway radar, beam 2 , between 00:00 UT and 06:00 UT on 10 December 2000 .

and the STARE data are all consistent with the interpretation of a poleward propagating upward FAC at around 03:52 UT. Other oscillations earlier in this wavepacket also show the signatures of upwards FAC; however they do not appear to have optical counterparts. Consequently, it is difficult to determine whether this arc is related to the pulsation activity which exists throughout the 03:30-04:00 UT interval or is driven by a separate propagating field aligned current (FAC) system which crosses the MIRACLE network at the same time. The wavepacket between 04:45-06:10 UT seems to represent a more continous wavetrain and can be seen to contain a number of discrete spectral components. Figure 20 shows the unfiltered $H$-component power spectrum between 04:30-05:45 UT; clear latitude-independent discrete spectral peaks are apparent at around 1.9, 2.7, 3.7, and $4.6 \mathrm{mHz}$. Latitudinal amplitude maxima and phase changes consistent with the excitation of FLRs are seen for each spectral component (not shown). Figure 21 shows the frequencies and the corresponding modulus of the $m$-values for the wavepackets seen in Fig. 13 for the two discrete frequencies seen between 01:30-02:00 UT (cf. Fig. 14) and the four discrete frequencies seen between 04:45-06:10 UT (cf Fig. 20).

$m$-values were calculated using the $D$-component from the SOD-PEL station pair, to minimise the effects of latitudinal phase changes which dominate near the resonance and have more influence in the $H$-rather than the $D$-component. The $m$-values, shown in Fig. 21, were all measured to be negative (westward) consistent with tailward phase propagation in the morning sector; however, the figure shows the modulus of $m$ for clarity. The frequency components from the earlier wave packet are marked with an $x$-symbol in Fig. 21, the error bars in frequency and $m$-value are calculated on the basis of the bandwidth of the complex demodulation analysis used to isolate each spectral component and the standard deviation in the $m$-value estimates taken over the maximum $H$-component amplitude demodulates, respectively. Both wavepackets suggest that there might be an almost linear relationship between frequency and $m$-value; the dot-dashed lines show an approximate best-fit line through the origin and the data points. Hence, we can estimate the azimuthal phase speed of these wavepackets in the ionosphere, using the relationship $v_{p h I}=2 \pi R_{E} \cos \Lambda f / m$ where $R_{E}$ is the Earths radius, $\Lambda$ is the latitude of the observation and $f$ is the wave frequency. This results in (westward) ionospheric 
STARE/SuperDARN Hankasalmi 10 Dec 2000

(a) STARE beam 2 power, ranges 303234363840

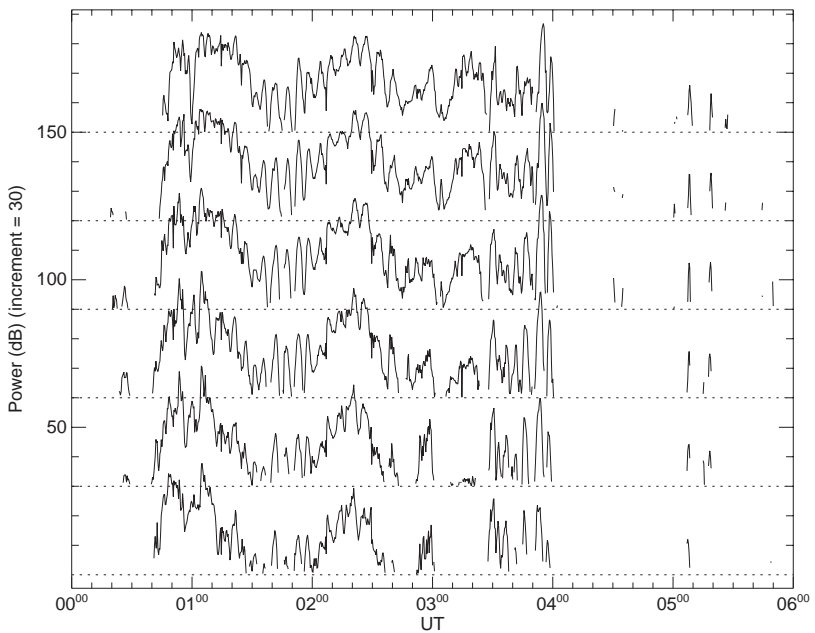

(b) Hankasalmi beam 5 velocity, ranges 182022

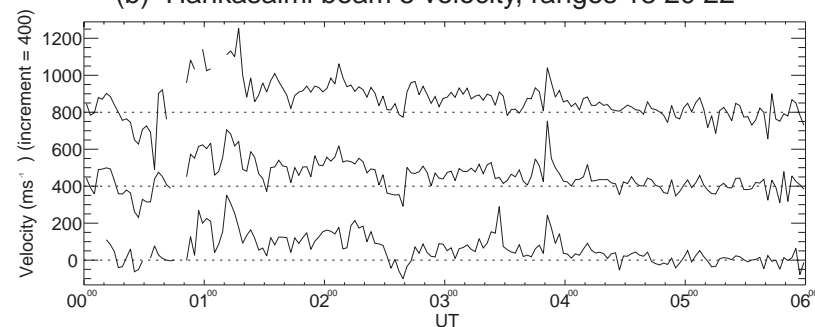

Fig. 17. Stacked STARE Norway radar backscatter power from beam 2 and range gates 30, 32, 34, 36, 38 and 40 (a), and stacked SuperDARN Hankasalmi radar beam 5 velocity from range gates 18, 20 and 22, between 00:00 UT and 06:00 UT on 10 December 2000 (b).

\section{STARE PARAMETER PLOT}

10 Dec 2000: STARE Norway beam 2 pwr

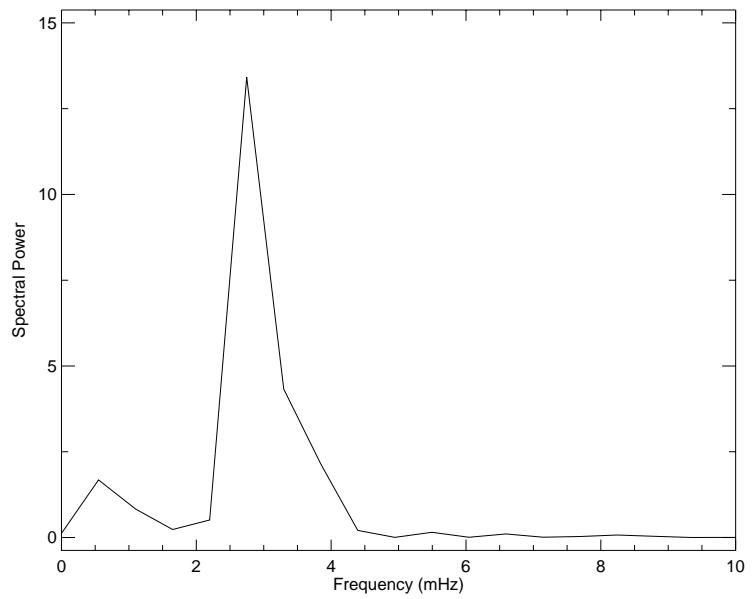

Fig. 18. Power spectrum from STARE Norway beam 2, range gate 38, between 01:30 UT and 02:00 UT.

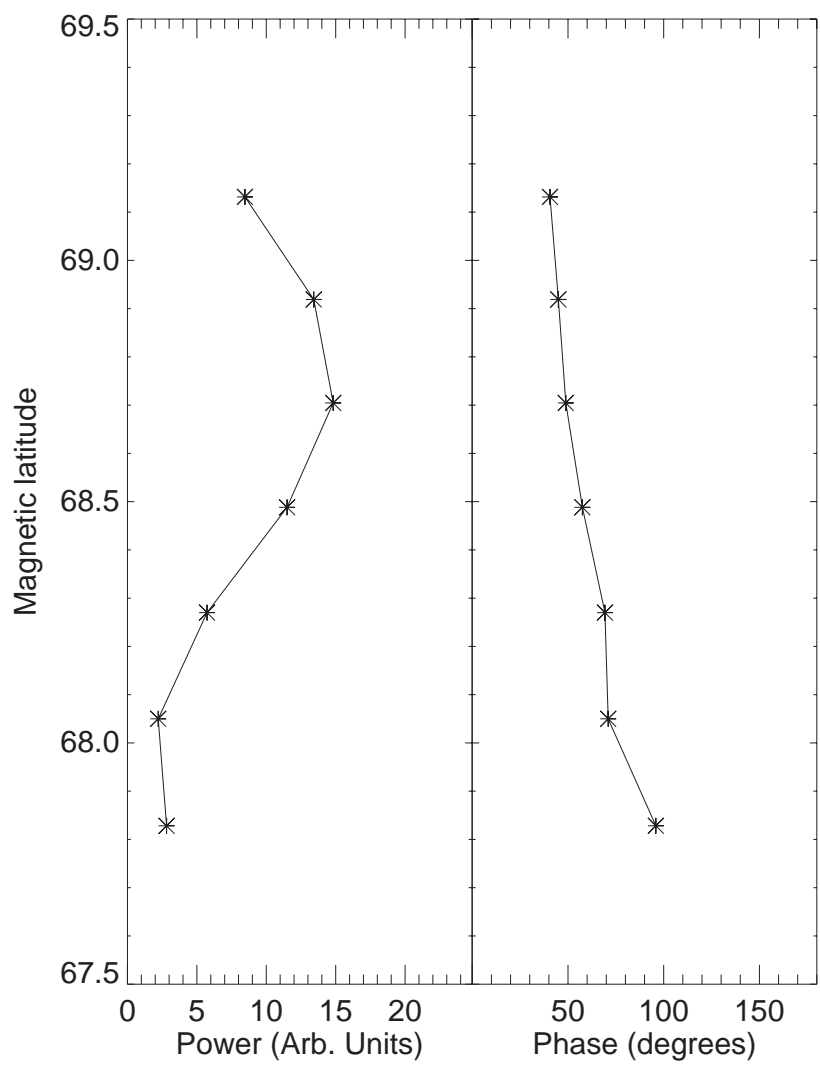

Fig. 19. Amplitude and phase of the $2.7 \mathrm{mHz}$ spectral component observed by the STARE Norway radar beam 2 in range gates 28, 30, $32,34,36,38,40$, in the interval 01:30-02:00 UT on 10 December 2000 .

phase speeds of $6.7 \mathrm{~km} / \mathrm{s}$ and $12.0 \mathrm{~km} / \mathrm{s}$ for each of the two wavepackets respectively, where we have assumed a latitude of $66^{\circ}$ close to the stations recording maximum amplitudes. These phase speeds compare well with previous estimates of FLR common azimuthal phase speeds as presented, for example, by Mathie and Mann (2000) (e.g. their Fig. 4), and are consistent with the downtail (westward) phase propagation expected in the morning sector.

\section{Discussion}

The characteristics of the FLRs reported here are in good agreement with those recently presented by previous authors who have attributed the FLR excitation to waveguide modes (e.g. Mathie et al., 1999). Recent theoretical work by Mann et al. (1999) (see also Mills et al., 1999) has shown that the Kelvin-Helmholtz instability can excite body type waveguide modes, in addition to the standard KH surface waves. Later work by Mills and Wright (1999) and Mann and Wright (1999) (see also Wright and Rickard, 1995) has shown that multiple FLRs excited by several radial waveguide mode harmonics will be expected to possess the same azimuthal phase speed. Observational evidence (e.g. Mathie and Mann, 2000) has now placed this hypothesis on a firmer footing by show- 


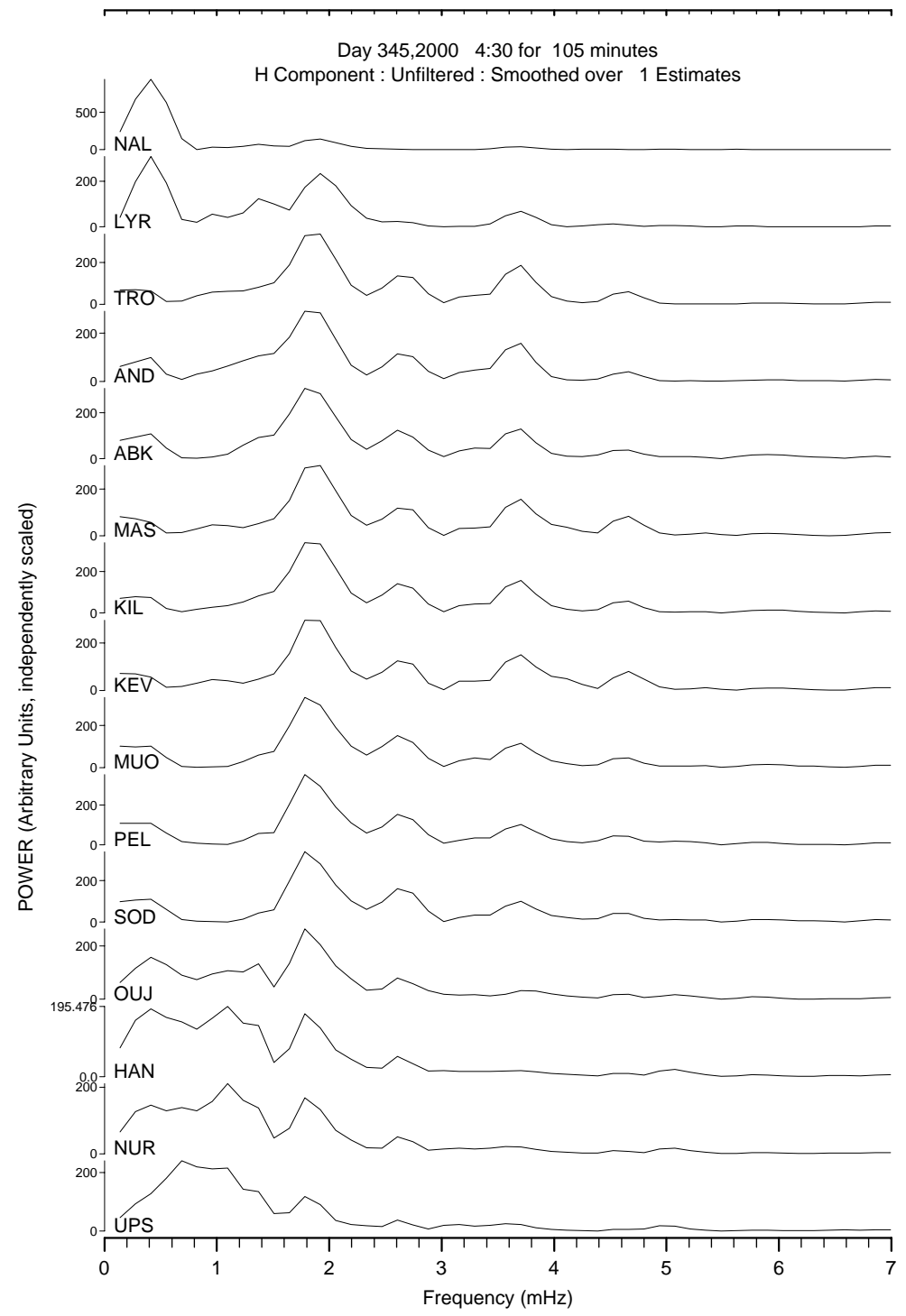

Fig. 20. Unfiltered $H$-component power spectra from selected IMAGE magnetometer stations between 04:3005:45 UT. Clear latitude independent spectral peaks are apparent at around 1.9, 2.7, 3.7 and $4.6 \mathrm{mHz}$.

ing clearly how common azimuthal phase speed FLRs can be driven during fast solar wind speed intervals. According to the theory of Mann et al. (1999), body waveguide modes (in addition to the usual surface modes) can be energised by the KHI once the magnetosheath flow speed exceeds a critical speed $\sim 500 \mathrm{kms}^{-1}$ (the exact critical speed depends upon variations in the sound and Alfvén speeds and the magnetic field across the magnetopause). The theory is in excellent agreement with the observations of Engebretson et al. (1998) (see also Mathie and Mann, 2001) who showed that ULF wave power in the morning side increases significantly when the solar wind speed $\gtrsim 500 \mathrm{kms}^{-1}$. Statistical studies of the diurnal variation of Pc5 ULF wave power show that it is strongly peaked in the morning local time sector. As discussed by Mann and Wright (1999), stabilising IMF magnetic field tension in the dusk local time sector is usually cited as a likely reason for the dawn-dusk flank asymmetry (see Lee and Olson, 1980). Further, and as shown by Mann et al. (1999), the KHI acts through the propagation of disturbances against the background flow. In the magnetospheric frame, the down-tail phase speed of waves driven by the KHI only occurs because they are advected downstream by the flow. This should result in azimuthal phase speeds, $v_{p h}$, for KH driven FLRs which are $<U$.

In the observations we have presented here, there is clear observational evidence for the excitation of large amplitude Pc5 FLRs on both the dawn and dusk flanks during an interval of fast solar wind speed. Figure 21 shows that the multiple frequency FLRs observed on the dawn flank in the intervals between 01:30-02:00 UT and 04:45-06:10 UT had common ionospheric azimuthal phase speeds of $6.7 \mathrm{kms}^{-1}$ and $12.0 \mathrm{kms}^{-1}$, respectively. Assuming an equatorial magnetopause standoff distance of $10 R_{E}$, produces estimates of the magnetopause phase speeds of around $170 \mathrm{kms}^{-1}$ and $300 \mathrm{kms}^{-1}$, respectively. These phase speeds are likely to be less than $U$ at the local times of the measurements, espe- 


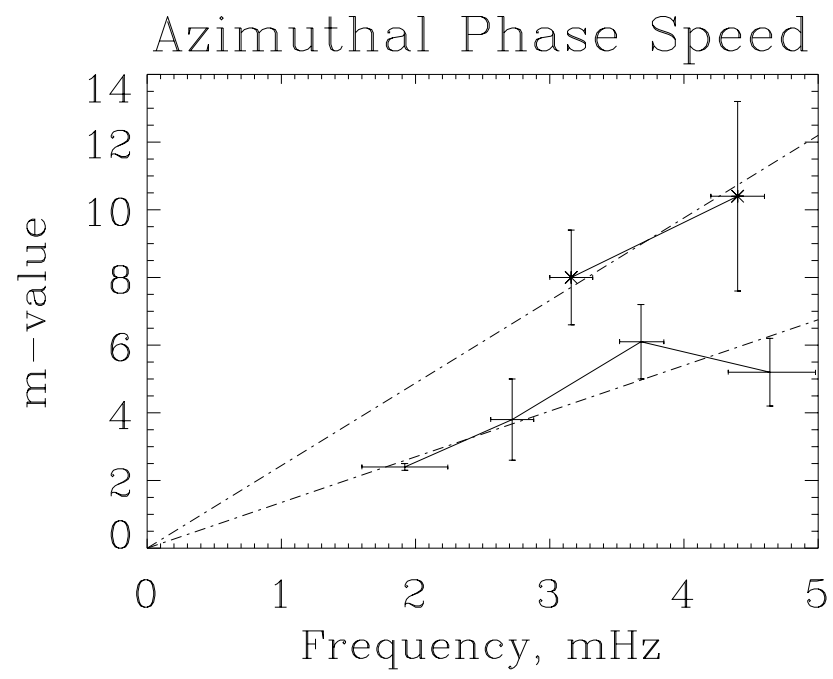

Fig. 21. Wave frequency and $m$-values from the two discrete frequencies in the wavepacket between 01:30-02:00 UT ( $x$-symbols) and the four discrete frequencies in the wavepacket between 04:4506:10 UT. Dot-dashed lines represent lines of approximate best fit through the origin in each case. See text for more details.

cially given that on the flanks the magnetosheath speed approaches the upstream solar wind speed (e.g. Spreiter and Stahara, 1980). Consequently, the low, common azimuthal phase speed property of the FLRs on the dawn flank provides excellent evidence that the KHI was responsible for energising waveguide modes which themselves drive large amplitude FLRs on the dawn flank (cf. Mann and Wright, 1999).

On the dusk flank, however, multiple frequency FLRs were not recorded in the interval studied (between 00:0000:40 UT). Using the $m$-value estimated from the $D$ component at the ISL and MCM stations $\left(\Lambda \approx 64.5^{\circ}\right)$, for a 6 min period wave, results in a significantly faster ionospheric azimuthal phase speed of around $28 \mathrm{kms}^{-1}$. Mapping this speed to a model magnetopause at $10 R_{E}$ produces a magnetopause phase speed $\sim 650 \mathrm{kms}^{-1}$, very similar to the upstream solar wind speed. As discussed above, the fastest growing KHI driven modes will, in general, be expected to drive FLRs with phase speeds somewhat lower than the local sheath speed (see e.g. Mills and Wright, 1999, their Fig. 11); however, more slowly growing modes might be excited with speeds closer to $U$. Hence, on the basis of the phase speed, we cannot unambigously determine whether the dusk flank FLR was driven by a KH unstable waveguide mode. Indeed, according to Mann and Wright (1999), a phase speed $v_{p h} \sim U$ may be more suggestive of a magnetopause running pulse driver than the development of the KHI.

Very recently, Sarafopoulos et al. (2001) have presented ground-based magnetometer observations of large amplitude ULF waves on both the dawn and dusk flanks during an interval of fast solar wind speed. They also proposed the $\mathrm{KHI}$ as the mechanism which energised the pulsations. Despite having no in situ measurements local to the magne- topause, Sarafopoulos et al. (2001) observed periodic variations in the $>38 \mathrm{keV}$ electron channel on Geotail in the magnetosheath. These authors argued that magnetopause motion provided a periodic magnetic connectivity between Geotail and the oscillating magnetopause. The electron signatures occurred during intervals of magnetic connectivity to the magnetopause, providing a remote signature of periodic magnetopause oscillations on the dawn flank.

In this study, we are fortunate to have in-situ Cluster data from the dusk flank which can be used to examine the motion and orientation of the magnetopause and boundary layers. The Cluster data can be used to test the hypothesis that the ground pulsations are related to global waveguide modes with corresponding periodic magnetopause motion, perhaps due to the excitation of the KHI. During the interval immediately preceding the observations of the largest amplitude ULF waves on the ground in the Canadian sector (00:00-00:40 UT), the Cluster satellites were following an outbound trajectory through the high latitude dusk lobe and into the magnetosheath, crossing the MP at 23:40 UT. Following the entry into the magnetosheath BL, at 22:10 UT, Cluster repeatedly and quasi-periodically sampled regions of low $|\boldsymbol{B}|$ in the magnetosheath boundary layer (BL) between 22:24-22:56 UT (and possibly longer until around 23:16 UT). While the location of the Cluster quartet in relation to the equilibrium position of the magnetopause was not such that motion of the magnetopause and BL caused Cluster to repeatedly cross the magnetopause, the Cluster observations were consistent with the quasi-periodic motion advecting a locally planar magnetosheath $\mathrm{BL}$ across the satellites. This might have resulted in the repeated observation of regions of lower magnetic field magnitude as the satellites repeatedly made observations closer to the magnetopause.

As discussed by Fairfield et al. (2000) (see also Otto and Fairfield, 2000, and references therein), for MP crossings which move satellites into the magnetosheath from the magnetosphere, the action of the KHI might be expected to rotate the MVA magnetopause normal in a sunward direction as compared to the expected model MP orientation. While the periodic motion in the magnetosheath boundary layer did not generate multiple magnetopause crossings, when Cluster crossed the MP at 23:40 UT a slight sunward rotation of the normal was observed which may be consistent with the action of the $\mathrm{KH}$ in the manner described by Fairfield et al. (2000).

Interestingly, for the quasi-periodic inward motion of the magnetosheath BL (which moves the Cluster satellites further towards the magnetopause) the 4 satellite timings are consistent with the motion of a locally planar structure which is approximately aligned with the expected local MP orientation. If the flank waveguide has a magnetopause standoff distance of $10 R_{E}$, typical of waveguide mode driven FLRs with azimuthal wavenumbers $m \sim 2-10$ will have downtail (azimuthal) wavelengths $\sim 6-31 R_{E}$. These wavelengths are very much larger than likely ampitudes of magnetopause displacement due to the KHI. Consequently, the angular distortion of the magnetopause normal detected 
for outbound (magnetosphere to magnetosheath) crossings produced by these long-wavelength $\mathrm{KH}$ driven waveguide modes would probably be quite small. This appears to be consistent with the hypothesis that the quasi-periodic structures seen by Cluster might be related to magnetopause motion from $\mathrm{KH}$ driven waveguide modes.

While the inward motion of the BL appears to be wellordered by the expected magnetopause orientation, for the outwards motion of the BL (effectively inbound motion of Cluster towards the magnetosphere) the inter-satellite timings and inferred boundary orientations appear to be much more complex. Indeed, in general these do not seem to be clearly ordered by their timings in relation to the expected model magnetopause orientation. This suggests that, if the KHI was responsible for the motion of the magnetosheath $\mathrm{BL}$, it may have developed some vortex structure which is asymmetric with respect to inward and outward motion through the magnetosheath BL. Indeed, in their recent papers Fairfield et al. (2000) and Otto and Fairfield (2000) have presented evidence from MHD simulations and Geotail observations that KHI vortices can produce distortion of the leading edge of the vortex which is seen as a crossing from the magnetosheath into the magnetosphere (inbound in relation to the satellite), as compared to a well defined crossing which is observed in the trailing edge when the satellites cross from the magnetosphere into the magnetosheath (outbound). This results in asymmetries between outbound and inbound crossings such that only the magnetosphere to magnetosheath crossings produce well-ordered normals which are rotated slightly sunwards from the expected model MP normal orientation.

Recent observations of 39 encounters with the magnetopause, as measured by the Cluster Electric Field and Wave Experiment and presented by S. D. Bale et al. ["The normal, thickness, and speed of the dusk magnetopause current layer from Cluster Electric Field and Wave experiment measurements of spacecraft potential", unpublished manuscript, 2001] have also provided further clear evidence that magnetosheath to magnetosphere crossings are much less wellordered by the expected magnetopause orientation, are more dispersed and less coherent between the spacecraft than magnetosphere to magnetosheath crossings. If the same asymmetry of distorted planar geometries is generated by the KHI within the BL, as might be expected, then identical inbound/outbound asymmetry might be expected even during intervals when Cluster does not actually cross the MP. This appears to be in accord with the observations reported here and may provide further evidence that Cluster was observing the effects of $\mathrm{KHI}$ on the magnetopause in the magnetosheath BL. As shown by Otto and Fairfield (2000), during times when the satellites are monitoring regions close to a $\mathrm{KH}$ vortex, the magetic field in the $X-Y$ plane is likely to be distorted by the field of the KHI. The fact that the entries into regions of low $|\boldsymbol{B}|$ presented here were also characterised by changes in $B_{X}$ and $B_{Y}$ may also be supportive of the action of the KHI (cf. the Geotail observations presented by Fairfield et al., 2000).
In a recent study, Wright et al. (2000) considered the nature of the waveguide modes which might be excited by the KHI. They concluded that, while KH surface waves are likely to become strongly non-linear on the flanks, $\mathrm{KH}$ driven body waveguide modes have much longer growth lengths along the magnetopause and are likely to develop to a much less advanced non-linear stage. Wright et al. (2000) show that the $\mathrm{KH}$ driven body modes are only convectively unstable and have down-tail growth lengths $\sim 20 R_{E}$; they suggested that this could explain the success of linear models in reproducing the observed characteristics of waveguide mode driven FLRs. Our observations suggest that some vortex features may have developed at the leading edge of the magnetopause in $\mathrm{KH}$ driven waveguide modes, however, given the results from the Wright et al. (2000) study, any vortex features which develop would only be likely to reach a fairly weakly non-linear stage.

The observations we have presented from ground-based instruments in the dusk sector also provide confirmation of the close link between field line resonances and the discrete auroral arcs. Ground-based radar, magnetometer and optical data all showed clear evidence of the latitudinal amplitude and phase characteristics expected of a well-developed FLR, including phase mixing signatures and poleward phase propagation. Figure 5, in particular, illustrates the very close relationship between the electron precipitation and the FLR fields. Electron precipitation occurs on latitudes near the poleward boundary of the resonance region which itself is close to the open-closed field line separatrix. This implies that large amplitude field line resonances may excite electron precipitation on stretched magnetic field lines (e.g. Greenwald and Walker, 1980; Samson et al., 1991, 1996). Moreover, both the oscillations of luminosity measured by the MSP (Fig. 5) and the pulses of the azimuthal convection flow (Fig. 8) are seen only during the later stages of the field line resonance evolution. Indeed, the largest increases in luminosity are only observed once the amplitude of the FLR has started to decay, presumably due to the action of ionospheric dissipation once the fast mode is no longer driving the FLR, perhaps through propagation down the waveguide (see, for example Wright, 1994; Rickard and Wright, 1994, 1995). This suggests that there may be a finite time between the excitation of the FLR and its triggering of auroral precipitation.

In several studies, it has been suggested that FLRs may accelerate electrons in discrete arcs once the perpendicular FLR scales reach electron inertial length scales in the auroral acceleration region (e.g. Streltsov and Lotko, 1997). As discussed by Mann et al. (1995) and Mann (1997) (see also Wright and Allan, 1996b), for an evolving FLR these electron inertial scales can only be reached after a finite time and they usually require large local background perpendicular Alfvén speed gradients. Consequently, one might expect that FLRs may only generate parallel electric fields after a finite time once the fields have phase mixed to inertial length scales. In Fig. 5, the dominant optical emissions in the 00:00-00:40 UT wavepacket occur late in the wave packet, which may be supportive of the conclusion that the FLR 
fields must evolve and phase mix to sufficiently fine scales before significant parallel electron acceleration can occur. Similarly, the arc motions seen by MIRACLE, on the dawn flank in the European sector, were only seen at the end of the 03:30-04:00 UT wavepacket.

To our knowledge, this is the first time that FLR signatures have been seen simultaneously and at the same location with radar, magnetometer and optical instrumentation; earlier studies such as that presented by Samson et al. (1996) have shown characteristics in these three instruments; however, they were not measured at the exactly the same location nor at exactly the same time. Consequently, our observations provide further clear evidence in support of a strong, possibly causal relationship between FLRs and some types of discrete auroral arc.

\section{Summary}

We have presented co-ordinated ground-based and Cluster observations which demonstrate that very large amplitude Pc5 ULF waves can be excited on the flanks of the magnetosphere during fast solar wind speed intervals. These global scale ULF waves possess discrete frequency characteristics which are consistent with the energising of magnetospheric waveguide modes and subsequently FLRs. Cluster observed quasi-periodic motion of the magnetosheath boundary layer (BL) and ULF pulsations of the same period observed on the ground provide further support for the waveguide mode paradigm by demonstrating the periodic boundary motion expected for a global magnetospheric waveguide mode.

Our observations support the hypothesis that a long azimuthal wavelength $\mathrm{KH}$ instablity developed on the dusk flank magnetopause (MP) and that the consequent advection of plasma in the magnetosheath boundary layer caused Cluster to periodically sample regions lying deeper in the magnetosheath. The fact that the frequency of the quasiperiodic Cluster entries deeper into the magnetosheath $\mathrm{BL}$ closely matched the period of the ULF waves observed on the ground strongly supports the hypothesis that the pulsations observed on the ground were related these magnetopause fluctuations. Moreover, an apparent asymmetry observed between inbound and outbound motion of the magnetosheath $\mathrm{BL}$ adds credence to the hypothesis that magnetopause KHI acted as the energy source for the excitation of the global scale waveguide modes. Given that multiple discrete frequency FLRs were also observed on the dawn-side, with common azimuthal phase speeds which were less than the expected magnetosheath speed, our observations also suggest that the KHI was simultaneously energising waveguide modes and FLRs on the dawn flank.

Using co-incident observations from ground-based magnetometer, optical and radar instrumentation we also presented the first evidence from co-incident measurements of a very close relationship between FLR fields and the generation of discrete auroral arcs. Our observations demonstrate that the excitation of ULF waves in the form of magnetospheric waveguide modes may provide an important solar wind-magnetosphere coupling mechanism, as well as possibly being reponsible for important magnetosphereionosphere coupling via the generation and modulation of electron precipitation into the ionosphere.

Despite the fact that ULF waves were observed on both flanks in this study, statistical studies show that, in general, Pc5 ULF wave power peaks in the morning local time sector. This is consistent with the preferential action of the KHI on the dawn flank, perhaps as a result of IMF tension stabilising the KHI on the dusk side. Since the Cluster orbit is precessing from dusk, past noon and towards dawn, there are likely to be other interesting conjunctions between the Cluster satellites and ground-based instrumentation in the morning sector. These conjunctions should allow more detailed co-ordinated ground-satellite studies to be completed in the future. Finding intervals when Cluster repeatedly enters and exits the magnetopause during periods of fast solar wind speed will allow the importance of the KHI for driving global scale ULF waves to be more fully determined. These studies may provide further evidence of the importance of magnetospheric waveguide modes and FLRs as a pathway for energy transport in solar wind-magnetosphere-ionosphere coupling.

Acknowledgements. We thank David McComas and Ruth Skoug for providing level 2 SWEPAM data from ACE. The IMAGE magnetometer data are collected as a Finnish-German-NorwegianPolish-Russian-Swedish Project. The CANOPUS programme is a project of the Canadian Space Agency. SAMNET is a UK PPARC National Facility and is operated and deployed by the University of York. The CUTLASS HF radars are deployed and operated by the University of Leicester and are funded as a UK National facility by the Particle Physics and Astronomy Research Council (Grant no. PPA/R/R/1997/00256).

Topical Editor G. Chanteur thanks A. D. M. Walker and G. Belmont for their help in evaluating this paper.

\section{References}

Baker, K. B. and Wing, S.: A new magnetic co-ordinate system for conjugate studies at high-latitudes, J. Geophys. Res., 94, 9139, 1989.

Balogh, A., Carr, C. M., Acuna, M. H., Dunlop, M. W., Beek, T. J., Brown, P., Fornacon, K.-H., Georgescu, E., Glassmeier, K.-H., Harris, J., Musmann, G., Oddy, T., and Schwingenschuh, K.: The Cluster Magnetic Field Investigation: Overview of in-flight performance and initial results, Ann. Geophysicae, 19, 1207-1217, 2001.

Beamish, D., Hanson, H., and Webb, D.: Complex demodulation applied to Pi2 geomagnetic pulsations, Geophys. J. R. Astron. Soc., 58, 471, 1979.

Blanchard, G. T., Lyons, L. R., and Samson, J. C.: Accuracy of using $6300 \AA$ auroral emission to identify the magnetic separatrix on the nightside of Earth, J. Geophys. Res., 102, 9697, 1997.

Chen, L. and Hasegawa, A.: A theory of long-period magnetic pulsations, 1, Steady state excitation of field line resonance , J. Geophys. Res., 79, 1033, 1974.

Dunlop, M. W., Balogh, A., Baumjohann, W., Haerendel, G., Fornacon, K.-H., and Georgescu, K.-H., et al.: Dynamics and lo- 
cal boundary properties of the dawn-side magnetopause under conditions observed by Equator-S, Ann. Geophysicae, 17, 1535, 1999.

Dungey, J. W.: Electrodynamics of the outer atmosphere, Proceedings of the Ionosphere, Phys. Soc. of London, London, p. 255, 1955.

Engebretson, M., Glassmeier, K.-H., Stellmacher, M., Hughes, W. J., and Lühr, H.: The dependence of high-latitude Pc5 wave power on solar wind velocity and on the phase of high-speed solar wind streams, J. Geophys. Res., 103, 26 271, 1998.

Fairfield, D. H., Otto, A., Mukai, T., Kokubun, S., Lepping, R. P., Steinberg, J. T., Lazarus, A. J., and Yamamoto, T.: Geotail observations of the Kelvin-Helmholtz instability at the equatorial magnetotail boundary for paralled northwards fields, J. Geophys. Res., 105, 21 159, 2000.

Fejer, B. G. and Kelly, M. C.: Ionospheric irregularities, Rev. Geophys. Space Phys., 18, 401, 1980.

Fenrich, F. R., Samson, J. C., Sofko, G., and Greenwald, R. A.: ULF high- and low- $m$ field line resonances observed with the Super Dual Auroral Radar Network, J. Geophys. Res., 100, 21 535, 1995.

Greenwald, R. A., and Walker, A. D. M.: Energetics of longperiod resonant hydromagnetic waves, Geophys. Res. Lett., 7, $745,1980$.

Greenwald, R. A., Baker, K. B., Dudeney, J. R., Pinnock, M., Jones, T. B., Thomas, E. C., Villain, J.-P., Ceriser, J.-C., Senior, C., Hanoise, C., Hunsucker, R. D., Sofko, G., Koehler, J., Nielsen, E., Pellinan, R., Walker, A. D. M., Sato, N., and Yamagishi, H.: DARN/SuperDARN: A global view of the dynamics of highlatitude convection, Space Sci. Rev., 71, 761, 1995.

Hughes, W. J. and Southwood, D. J.: The screening of micropulation signals by the atmosphere and ionosphere, J. Geophys. Res., 81, 3234, 1976.

Kivelson, M. G. Etcheto, J., and Trotignon, J. G.: Global Compressional Oscillations of the Terrestrial Magnetosphere: The Evidence and a Model, J. Geophys. Res., 89, 9851, 1984.

Kivelson, M. G. and Southwood, D. J.: Resonant ULF waves: A new interpretation, J. Geophys. Res., 12, 49, 1985.

Kivelson, M. G. and Southwood, D. J.: Coupling of global magnetospheric MHD eigenmodes to field line resonances, J. Geophys. Res., 91, 4345, 1986.

Lee, L.C. and Olson, J. V.: Kelvin-Helmholtz instability and the variation of geomagnetic pulsation activity, J. Geophys. Res., 7, 777,1980

Lühr, H., Aylward, A., Buchert, S. C., Pajunpää, A., Pajunpää, K., Holmboe, T., and Zalewski, S. M.: Westward moving dynamic substorm features observed with the IMAGE magnetometer network and other ground-based instruments, Ann. Geophysicae, 16, 425, 1998.

Mann, I. R., Wright, A. N., and Cally, P. S.: Coupling of magnetospheric cavity modes to field line resonances: A study of resonance widths, J. Geophys. Res., 100, 19441, 1995.

Mann, I. R.: On the internal radial structure of field line resonances, J. Geophys. Res., 102, 27 109, 1997.

Mann, I. R., Chisham, G., and Bale, S. D.: Multi-satellite and ground-based observations of a tailward propagating Pc5 magnetospheric waveguide mode, J. Geophys. Res., 103, 4657, 1998.

Mann, I. R., Wright, A. N., Mills, K. J., and Nakariakov, V. M.: Excitation of magnetospheric waveguide modes by magnetosheath flows, J. Geophys. Res., 104, 333, 1999.

Mann, I. R. and Wright, A. N.: Diagnosing Pc5 magnetospheric waveguide mode excitation mechanisms, Geophys. Res. Lett.,
26, 2609, 1999.

Mathie, R. A. and Mann, I. R.: Observations of harmonic Pc5 field line resonance phase speeds: A diagnostic of their excitation mechanism, J. Geophys. Res., 105, 10713, 2000.

Mathie, R. A. and Mann, I. R.: On the solar wind control of Pc5 ULF pulsation power at mid-latitudes: Implications for $\mathrm{MeV}$ electron acceleration in the outer radiation belt, J. Geophys. Res., 106, 29783, 2001

Mathie, R. A., Mann, I. R., Menk, F. W., and Orr, D.: Pc5 ULF pulsations associated with waveguide modes observed with the IMAGE magnetometer array, J. Geophys. Res., 104, 7025, 1999.

McDiarmid, D. R., Yeoman, T. K., Grant, I. F., and Allan, W. Simultaneous observations of traveling vortex structure in the morning sector and a field line resonance in the post-noon sector, J. Geophys. Res., 99, 8891, 1994.

McDiarmid, D. R., Wright, A. N., and Allan, W.: Time-limited excitation of damped field-line resonances: Implications for satellite observations, J. Geophys. Res., 104, 17 409, 1999.

McComas, D. J., Bame, S. J., Barker, P., Feldman, W. C., Phillips, J. L., and Riley, P.: Solar wind electron, proton, alpha monitor, Space Sci. Rev., 86, 563, 1998.

Mills K., Wright , A. N., and Mann, I. R.: Kelvin-Helmholtz driven modes of the magnetosphere, Phys. Plasmas, 6, 4070, 1999.

Mills K. and Wright, A. N.: Azimuthal phase speeds of field line resonances driven by Kelvin-Helmholtz unstasble waveguide modes, J. Geophys. Res., 104, 22 667, 1999.

Otto, A. and Fairfield, D. H.: Kelvin-Helmholtz instability at the magnetotail boundary: MHD simulation and comparison with Geotail observations, J. Geophys. Res., 105, 21 175, 2000.

Rickard, G. J. and Wright, A. N.: Alfvén resonance excitation and fast-wave propagation in magnetospheric waveguides, J. Geophys. Res., 99, 13 455, 1994.

Rickard, G. J. and Wright, A. N.: ULF pulsations in a magnetospheric waveguide - Comparison of real and simulated satellite data, J. Geophys. Res., 100, 3531, 1995.

Robinson, T. R.: Towards a self-consistent non-linear theory of radar auroral backscatter, J. Atmos. Terr. Phys., 48, 417, 1986.

Rostoker, G., Samson, J. C., Creutzberg, F., Hughes, T. J., McDiarmid, D. R., McNamara, A. G., Vallance Jones, A., Wallis, D. D., and Cogger, L. L.: CANOPUS - A ground-based instrument array for remote-sensing the high-latitude ionosphere during the ISTP/GGS program, Space Sci. Rev., 71, 743, 1995.

Ruohoniemi, J. M., Greenwald, R. A., Baker, K. B., and Samson, J. C.: HF radar observations of field line resonances in the midnight/early morning sector, J. Geophys. Res., 96, 15 697, 1991.

Samson, J. C., Hughes, T. J., Creutzberg, F., Wallis, D. D., Greenwald, R. A., and Ruohoniemi, J. M.: Observations of a detached, discrete arc in association with field line resonances, J. Geophys. Res., 96, 15 683, 1991.

Samson, J. C., Harrold, B. G., Ruohoniemi, J. M., and Walker, A. D. M.: Field line resonances associated with MHD waveguides in the magnetosphere, Geophys. Res. Lett., 19, 441, 1992.

Samson, J. C., Cogger, L. L., and Pao, Q.: Observations of field line resonances, auroral arcs and auroral vortex structures, J. Geophys. Res., 101, 17 373, 1996.

Samson, J. C., Rankin, R., and Voronkov, I.: Field line resonances, auroral arcs, and substorm intensifications , in: Geospace Mass and Energy Flow: Results From the International SolarTerrestrial Physics Program, Geophys. Monogr. Ser., (Eds) Horwitz, J. L., et al., 104, p. 161, AGU, Washington, D. C., 1998.

Sarafopoulos, D. V., Takahashi, K., and McEntire, R. W.: Periodic variations of magnetosheath energetic electron flux associated 
with global Pc5 pulsations, J. Geophys. Res., 106, 13 037, 2001.

Shand, B. A., Lester, M., and Yeoman, T. K.: The relationship between VHF radar aurora backscatter amplitude and irregularity phase speed: A statistical Study, Ann. Geophysicae, 14, 803, 1996.

Sonnerup, B. U. O. and Cahill, L. J.: Magnetopause structure and attitude from Explorer 12 observations, J. Geophys. Res., 72, $171,1967$.

Southwood, D. J.: Some features of field line resonances in the magnetosphere, Planet. Space Sci., 22, 483, 1974.

Spreiter, J. R. and Stahara, S. S.: A new predictive model for determining solar wind-terrestrial planet interactions, J. Geophys. Res., 85, 6769, 1980.

Streltsov, A. V. and Lotko, W.: Dispersive, non-radiative field line resonances in a dipolar magnetic field geometry, J. Geophys. Res., 102, 27 121, 1997.

Syrjäsuo, M. T., Pulkkinen, T. I., Janhunen, P., Viljanen, A., Pellinan, R. J., Kauristie, K., Opgenoorth, H. J., Wallman, S., Eglitis, P., Karlsson, P., Amm, O., Nielsen, E., and Thomas, C.: Observations of substorm electrodynamics using the MIRACLE network, in: Substorms-4, (Eds) Kokubun, S. and Kamide, Y., Proc. International Conference on Substorms-4, Lake Hamana, Japan, Terra Scientific Publishing Company, Tokyo, 1998.

Tsyganenko, N. A.: Modelling the Earth's magnetospheric magnetic field confined within a realistic magnetopause, J. Geophys. Res., 100, 5599, 1996.

Voronkov, I., Friedrich, E., and Samson, J. C.: Dynamics of the substorm growth phase as observed using CANOPUS and SuperDARN instruments, J. Geophys. Res., 104, 1997.

Waldock, J. A., Jones, T. B., and Nielsen, E.: Statistics of 1-m wavelength plasma irregularities and convection in the auroral E-region, Radio Sci., 20, 709, 1985.

Walker, A. D. M., Ruohoniemi, J. M., Baker, K. B., and Greenwald, R. A.: Spatial and temporal behavior of ULF pulsations observed by the Goose Bay HF radar, J. Geophys. Res., 97, 12 187, 1992.

Wright, A. N.: Dispersion and wave coupling in inhomogeneous MHD waveguides, J. Geophys. Res., 99, 159, 1994.

Wright, A. N. and Allan, W.: Structure, phase motion and heating within Alfvén resonances, J. Geophys. Res., 101, 17 399, 1996a.

Wright, A. N. and Allan, W.: Are two-fluid effects relevant to ULF pulsations?, J. Geophys. Res., 101, 24 991, 1996 b.

Wright, A. N., Mills, K. J., Ruderman, M. S., and Brevdo, L.: The absolute and convective instability of the magnetospheric flanks, J. Geophys. Res., 105, 385, 2000.

Wright, A. N. and Rickard, G. J.: ULF pulsations driven by magnetopause motions: Azimuthal phase characteristics, J. Geophys. Res., 101, 24 991, 1995.

Yeoman, T. K., Milling, D. K., and Orr, D.: Pi2 pulsation polarisation patterns on the UK Sub-Auroral Magnetometer Network, Planet. Space Sci., 38, 589, 1990a.

Yeoman, T. K., Lester, M., Orr D., and Lühr, H.: Ionospheric boundary conditions of hydromagnetic waves: The Dependence on Azimuthal Wavenumber and a Case Study, Planet. Space Sci., 38, 1315, 1990b.

Yeoman, T. K., Tian, M., Lester, M., and Jones, T. B.: A Study of Pc5 Hydromagnetic Waves with Equatorward Phase Propagation, Planet. Space Sci., 40, 797, 1992.

Ziesolleck, C. W. S., Fenrich, F. R., Samson, J. C., and McDiarmid, D. R.: Pc5 field line resonance frequencies and structure Observed by SuperDARN and CANOPUS, J. Geophys. Res., 103, $11771,1998$. 